\title{
The multifrequency emission of Mrk 501
}

\section{From radio to $\mathrm{TeV}$ gamma-rays}

\author{
K. Katarzyński ${ }^{1,2}, \mathrm{H} . \mathrm{Sol}^{2}$, and A. Kus ${ }^{1}$ \\ 1 Toruń Centre for Astronomy, Nicolaus Copernicus University, ul. Gagarina 11, 87100 Toruń, Poland \\ 2 DAEC, UMR 8631 du CNRS, Observatoire de Paris-Meudon, 5 place J. Janssen, 92195 Meudon Cedex, France
}

Received 23 June 2000 / Accepted 14 December 2000

\begin{abstract}
We present two-epoch, multifrequency complete modeling of Mrk 501 broad band emission from the radio frequencies up to the very high gamma ray regime as observed during April 1997 outburst. To reproduce the X-ray and the gamma-ray radiation we assume an homogeneous Synchrotron-Self-Compton (SSC) model and approximate the electrons energy distribution by a broken power-law function with a sharp cut-off at high energy. We assume also spherical geometry of the emitting region (a blob) and uniform magnetic field. For this scenario we derive constrains on physical parameters obtained from the observable quantities and can reproduce very well the observed Very High Energy (VHE) spectra. We find that the two different high energy states cannot be explained by varying the particle energy distribution only. To obtain good spectral fits we have to change additionally one of the global parameters which describe the emitting blob: the magnetic field intensity $(B)$, the radius $\left(R_{\mathrm{b}}\right)$ or the Doppler factor $\left(\delta_{\mathrm{b}}\right)$. We use a model of an inhomogeneous conical jet to explain radiation from the low radio frequencies up to the ultraviolet. A part of the physical parameters of this jet can be constrained from our fits of the high energy emission. The remaining parameters are estimated independently from other observations. We assume that the blob is placed somewhere inside the jet, relatively close to the central engine. Within this "blobin-jet" scenario we analyze the effect of External Inverse-Compton (EIC) scattering of low energy synchrotron photons from the jet and thermal radiation surrounding the central engine. We show that EIC scattering can also very well explain VHE emission of Mrk 501 when external radiation is dominated by photons from the jet itself, which is an interesting alternative of the "blob-in-jet" scenario. Additional inverse-Compton scattering of relatively weak thermal external radiation cannot explain alone the spectra but can significantly modify them, especially in the sub-TeV range, in comparison to spectral shape generated only with SSC model. We also analyze the absorption effect due to pair-production inside the source, which appears almost negligible. The same effect in the Intergalactic Infrared Background (IIB) may introduce significant changes to the observed VHE spectra. In the infrared and optical parts of the spectrum, the contribution of the host galaxy is well reproduced by an elliptical galaxy evolution model. Three miniflares of Mrk 501 occurred in ten days between 7 and 16 April 1997 at the X-ray and gamma-ray frequencies. They can be well reproduced by simple variation of the break in the electron energy distribution function on time scale of a few hours, superposed to variation of the blob radius and of the particle density on time scale of about two days.
\end{abstract}

Key words. radiation mechanisms: nonthermal - galaxies: active - BL Lacertae objects: individual: Mrk 501

\section{Introduction}

A wealth of AGN gamma-ray emission models have been developed these last years, following the discovery by EGRET of about seventy GeV gamma-ray AGNs and the observation by ground-based Cerenkov telescopes of several TeV gamma-ray BL Lacs. The two main classes of scenario, namely leptonic and hadronic models, consider that gamma rays are produced somewhere at the base of

Send offprint requests to: K. Katarzyński, e-mail: kat@astro.uni.torun.pl the radio jet and are Doppler boosted. In leptonic models, gamma-rays are due to inverse-Compton scattering of lower energy photons by relativistic electrons in the jet. Hadronic models assume the presence of ultra-high energy protons which lead to gamma ray emission after interaction and decay of secondary particles. Several models can fit reasonably well the observed high-energy spectra, but a number of basic question still remain.

Leptonic models have been quite successful in explaining the high-energy spectra of blazars at different activity states (e.g. Ghisellini \& Madau 1996; 
Dermer \& Schlickeiser 1993; Sikora et al. 1994; Inoue \& Takahara 1996). The X-ray and gamma-ray data gathered during last years on Mrk 421 (e.g. Punch et al. 1992; Petry et al. 1996; Takahashi et al. 1996; Zweernik et al. 1997) and on Mrk 501 (e.g. Quinn et al. 1996; Samuelson et al. 1998; Aharonian et al. 1999; Kataoka et al. 1999), the two main BL Lac detected at TeV energies by groundbased Cerenkov telescopes, seems to favor leptonic models in BL Lac objects (Catanese \& Weekes, 1999), where the gamma rays are produced via an inverse-Compton mechanism by a population of relativistic electrons, which scatter soft photons up to the TeV range (e.g. Jones 1968; Blumenthal \& Gould 1970). However the nature of the scattered photons is not yet clearly identified. The most popular scenario is the synchrotron self-Compton (SSC) process, which considers that the scattered soft photons are in fact generated by the same electrons in the synchrotron emission mechanism (with peak in the X-ray range, e.g. Bednarek \& Protheroe 1997; Tavecchio et al. 1998, hereafter TMG). The scattering of additional lowenergy photons, coming from the accretion disk and from the clouds or other components of the jet itself, remain an open possibility as described by external inverse-Compton (EIC) models (e.g. Sikora et al. 1994).

Hadronic models also show good ability to explain high energy emission of blazars (e.g. Mannheim 1993; Mucke \& Protheroe 2000; Pohl \& Schlickeiser 2000; Dermer 1999). The hadronic models usually assume that most of the particle energy is accumulated initially in protons which produce $\mathrm{e}^{ \pm}$pairs by $p p$ or $p \gamma$ interactions. The pairs produce high energy photons in synchrotron or Compton processes. The high energy photons escape from the source or produce new pairs via $\gamma \gamma \rightarrow \mathrm{e}^{+} \mathrm{e}^{-}$interactions. The new pairs again generate new high energy photons etc., leading to proton-induced cascades (PIC, Mannheim 1993). Another possibility is to directly consider the synchrotron emission of extremely high energy protons (Aharonian 2000). Although quite interesting and promising for astroparticle physics, hadronic models still have to face some difficulties. To fit high energy spectra, they require primary protons of ultra high energy $\left(E>10^{19} \mathrm{eV}\right)$, but the nature of the proton acceleration zone is not yet clear. They often need dense targets to produce TeV flares, which may induce too high pair opacities to $\mathrm{TeV}$ gamma-rays in the emitting region (Coppi et al. 1993). In addition, basic version of hadronic scenario would predict that $\mathrm{TeV}$ gamma rays should vary more slowly than low energy gamma rays, which is not observed (Catanese \& Weekes 1999). These difficulties may be overcome in more sophisticated models but imply quite complicated physics.

Although the high energy emission of blazars has been widely analyzed by itself, little effort has been put up to now to simultaneously fit in details the spectra from the VHE radiation down to the lowest radio frequencies, while this approach may provide an interesting clue to the various constituents of the AGN. Long-term monitoring of BL Lac gamma-ray emitting objects at different energy ranges, from radio to the $\mathrm{TeV}$, are currently in progress.
They will provide a better description of the variability on various time scales, with the possibility to determine time delays between events observed at different frequencies, which then will be mandatory to better constrain the emission models. To develop the tools to interpret such kind of multiwavelength monitoring, we present here a modeling as simple as possible for the continuous emission from blazars jets over a wide range of the electromagnetic spectrum. We consider mainly synchrotron and inverseCompton emission mechanisms with the additional contribution of the host galaxy in the optical range. A basic "blob-in-jet" scenario, with a SSC emitting homogeneous plasma blob embedded in an emitting radio jet, is developed and applied to Mrk 501. This approach allows us to reproduce the observed spectra for two epochs during active state of the source and to analyze the temporal evolution, emphasizing different time scales likely involved in the activity process.

In Sect. 2 we describe different models which we use for spectral fits. The models include the SSC emission from a plasma blob at the jet base, the radio synchrotron radiation of a conical inhomogeneous jet, the contribution of external inverse-Compton scattering and the stellar emission of the host galaxy. We also consider absorption effects due to pair-production in the source and due to IIB. Constraints and correlations between the source physical parameters are discussed in Sect. 3. Section 4 presents the application of the models to Mrk 501. Some comments on the temporal evolution are also given in this section. We discuss and summarize our results in Sect. 5. The observational data which we have used were collected simultaneously in X-rays by the BeppoSAX satellite (Pian et al. 1998) and in gamma-rays by the ground based telescope CAT (Djannati-Atai et al. 1999). Non-simultaneous low energy measurements were gathered from the literature.

\section{Modeling the emission}

The Spectrum Energy Distribution (SED) of Mrk 501 shows two characteristic broad peaks in X-rays and gamma-rays. Most of the recent blazars models interpret them as a result of synchrotron and inverse-Compton radiation originating from the jet base. Photons for inverseCompton scattering may come from the synchrotron radiation of the same emitting volume (SSC) or from external sources of radiation (EIC) such as accretion disc, clouds or intercloud medium. An additional third characteristic peak appears in the SED of Mrk 501 between the infrared and ultraviolet frequencies. This feature is mainly due to the contamination by stars and dust emission. Their contribution is especially well visible in Mrk 501 because of the large shift of the synchrotron and inverse-Compton peaks towards high frequencies.

\subsection{Homogeneous SSC model}

The homogeneous synchrotron-self-Compton models have been successfully used by several authors to explain, 
reasonably well, the observed spectra of blazars from the optical up to the VHE range. We adopt such approach and assume a spherical geometry for the emitting region, filled by relativistic electrons with a uniform density in a uniform magnetic field. We describe the electrons energy distribution by a broken power-law function with a sharp cut-off:

$N_{\mathrm{e}}(\gamma)=\left\{\begin{array}{l}K_{1} \gamma^{-n_{1}}, \gamma_{\min } \leq \gamma \leq \gamma_{\mathrm{b}} \\ K_{2} \gamma^{-n_{2}}, \gamma_{\mathrm{b}}<\gamma \leq \gamma_{\mathrm{cut}} .\end{array}\right.$

In the above formula $\gamma=E / m c^{2}$ is the Lorentz factor and $K_{2}=K_{1} \gamma_{\mathrm{b}}^{n_{2}-n_{1}}$. More discussion about this assumption is given in Sect. 3.1. Detailed calculations of the electron energy evolution within the emitting region can provide more accurate solutions (Kirk et al. 1998). However, high energy flares are not yet fully characterized, therefore these solutions have many parameters which are difficult to constrain (e.g. inverse-Compton cooling rate, acceleration rate, escaping rate).

The synchrotron emission coefficient for any energy distribution of electrons $\left[\mathrm{erg} \mathrm{s}^{-1} \mathrm{~cm}^{-3} \mathrm{~Hz}^{-1}\right.$ sterad $\left.^{-1}\right]$ is given by:

$j_{\mathrm{s}}^{\prime}\left(\nu_{\mathrm{s}}^{\prime}\right)=\frac{1}{4 \pi} \int_{\gamma_{\min }}^{\gamma_{\text {cut }}} N_{\mathrm{e}}(\gamma) P_{\mathrm{e}}\left(\nu_{\mathrm{s}}^{\prime}, \gamma\right) \mathrm{d} \gamma$,

where $\nu_{\mathrm{s}}^{\prime}$ is the frequency in the emitting blob rest frame, and $P_{\mathrm{e}}\left(\nu_{\mathrm{s}}^{\prime}, \gamma\right)$ is the mean emission coefficient for a single electron [erg s${ }^{-1} \mathrm{~Hz}^{-1}$ sterad $^{-1}$ ] integrated over the isotropic distribution of pitch angles. In our calculations we use an approximation of this parameter (see Appendix A) which allows us to integrate analytically emission and absorption coefficients for the power law electrons spectrum.

The synchrotron intensity $\left[\mathrm{erg} \mathrm{s} \mathrm{s}^{-1} \mathrm{~cm}^{-2}\right.$ sterad $^{-1}$ $\left.\mathrm{Hz}^{-1}\right]$ in the source frame may be obtained by solving transfer equation for the spherical geometry. In the specific case where the local synchrotron emission coefficient is assumed uniform the solution is given by (e.g. Bloom \& Marscher 1996; Kataoka et al. 1999):

$I_{\mathrm{s}}^{\prime}\left(\nu_{\mathrm{s}}^{\prime}\right)=\frac{j_{\mathrm{s}}^{\prime}\left(\nu_{\mathrm{s}}^{\prime}\right)}{k^{\prime}\left(\nu_{\mathrm{s}}^{\prime}\right)}\left(1-\frac{2}{\tau^{2}}\left[1-\mathrm{e}^{-\tau}(\tau+1)\right]\right)$,

where $\tau=2 R_{\mathrm{b}} k^{\prime}\left(\nu_{\mathrm{s}}^{\prime}\right), R_{\mathrm{b}}$ is the radius of emitting region and $k^{\prime}(\nu)$ is the absorption coefficient $\left[\mathrm{cm}^{-1}\right.$ ] (e.g. Rybicki \& Lightman 1979) given by

$k^{\prime}\left(\nu_{\mathrm{s}}^{\prime}\right)=-\frac{1}{8 \pi m_{\mathrm{e}} \nu_{\mathrm{s}}^{\prime 2}} \int_{\gamma_{\min }}^{\gamma_{\text {cut }}} \gamma^{2} \frac{\mathrm{d}}{\mathrm{d} \gamma}\left[\frac{N_{\mathrm{e}}(\gamma)}{\gamma^{2}}\right] P_{\mathrm{e}}\left(\nu_{\mathrm{s}}^{\prime}, \gamma\right) \mathrm{d} \gamma$.

Inserting (A.5) and (A.7) into (3) provide us with a long but very simple expression describing the synchrotron spectrum of the blob in its rest frame.

The synchrotron luminosity in the source frame for spherical geometry is then simply given by

$L_{\mathrm{s}}^{\prime}\left(\nu_{\mathrm{s}}^{\prime}\right)=4 \pi^{2} R_{\mathrm{b}}^{2} I_{\mathrm{s}}^{\prime}\left(\nu_{\mathrm{s}}^{\prime}\right)$.

The emitting blob at the base of the blazar jet is believed to have a bulk relativistic motion towards the observer.
Using transformation of the intensity $I(\nu)=\delta_{\mathrm{b}}^{3} I^{\prime}\left(\nu^{\prime}\right)$ for the "Doppler boosting" effect we can write the observed flux density as:

$F_{\mathrm{s}}\left(\nu_{\mathrm{s}}^{\prime}\right)=\pi \frac{R_{\mathrm{b}}^{2}}{d_{1}^{2}} \delta_{\mathrm{b}}^{3}(1+z) I_{\mathrm{s}}^{\prime}\left(\nu_{\mathrm{s}}^{\prime}\right)$,

where $d_{1}$ is the luminosity distance, $z$ is the redshift and $\delta_{\mathrm{b}}=\left[\Gamma_{\mathrm{b}}(1-\beta \cos \theta)\right]^{-1}$ is the Doppler factor where $\Gamma_{\mathrm{b}}$ is the blob Lorentz factor, $\theta$ is the angle of the blob vector velocity to the line of sight and $\beta=v / c$. To fully switch to the observer's frame we have also to transform the frequency

$\nu_{\mathrm{s}}=\frac{\delta_{\mathrm{b}}}{1+z} \nu_{\mathrm{s}}^{\prime}$

To calculate self-Compton radiation we follow the approach of Inoue \& Takahara (1996). We assume a uniform synchrotron intensity in the whole radiation region, corrected for the fact that in reality it decreases along the blob radius (Gould 1979). This effect can be simplified by scaling the intensity of the central point of the blob by a factor $3 / 4$ (Kataoka et al. 1999). Thus the emission coefficient is given by:

$j_{\mathrm{c}}^{\prime}\left(\nu_{\mathrm{c}}^{\prime}\right)=\frac{h}{4 \pi} \epsilon_{\mathrm{c}}^{\prime} q\left(\epsilon_{\mathrm{c}}^{\prime}\right), \quad \epsilon_{\mathrm{s} / \mathrm{c}}^{\prime}=\frac{h}{m_{\mathrm{e}} c^{2}} \nu_{\mathrm{s} / \mathrm{c}}^{\prime}$,

where $q\left(\epsilon_{\mathrm{c}}^{\prime}\right)$ is the differential photon production rate:

$q\left(\epsilon_{\mathrm{c}}^{\prime}\right)=\int \mathrm{d} \epsilon_{\mathrm{s}}^{\prime} n\left(\epsilon_{\mathrm{s}}^{\prime}\right) \int \mathrm{d} \gamma N_{\mathrm{e}}(\gamma) C\left(\epsilon_{\mathrm{c}}^{\prime}, \gamma, \epsilon_{\mathrm{s}}^{\prime}\right)$.

$C\left(\epsilon_{\mathrm{c}}^{\prime}, \gamma, \epsilon_{\mathrm{s}}^{\prime}\right)$ is the Compton kernel given by Jones (1968):

$$
\begin{gathered}
C\left(\epsilon_{\mathrm{c}}^{\prime}, \gamma, \epsilon_{\mathrm{s}}^{\prime}\right)=\frac{2 \pi r_{\mathrm{e}}^{2} c}{\gamma^{2} \epsilon_{\mathrm{s}}^{\prime}}[2 \kappa \ln (\kappa)+(1+2 \kappa)(1-\kappa) \\
\left.\quad+\frac{\left(4 \epsilon_{\mathrm{s}}^{\prime} \gamma \kappa\right)^{2}}{2\left(1+4 \epsilon_{\mathrm{s}}^{\prime} \gamma \kappa\right)}(1-\kappa)\right], \\
\kappa=\frac{\epsilon_{\mathrm{c}}^{\prime}}{4 \epsilon_{\mathrm{s}}^{\prime} \gamma\left(\gamma-\epsilon_{\mathrm{c}}^{\prime}\right)}
\end{gathered}
$$

and $n\left(\epsilon_{\mathrm{s}}^{\prime}\right)$ is the number density of the synchrotron photons per energy interval described by:

$n\left(\epsilon_{\mathrm{s}}^{\prime}\right)=\frac{3}{4} \frac{4 \pi}{h c \epsilon_{\mathrm{s}}^{\prime}} \frac{j_{\mathrm{s}}^{\prime}\left(\nu_{\mathrm{s}}^{\prime}\right)}{k^{\prime}\left(\nu_{\mathrm{s}}^{\prime}\right)}\left(1-\exp \left(-k^{\prime}\left(\nu_{\mathrm{s}}^{\prime}\right) R_{\mathrm{b}}\right)\right)$.

Because of the allowed kinematic energy ranges for a given value of $\gamma$ and $\epsilon_{\mathrm{c}}^{\prime}$ Eq. (11) must be inserted into Eq. (9) under condition:

$\epsilon_{\mathrm{s}}^{\prime} \leq \epsilon_{\mathrm{c}}^{\prime} \leq \gamma \frac{4 \epsilon_{\mathrm{s}}^{\prime} \gamma}{1+4 \epsilon_{\mathrm{s}}^{\prime} \gamma}$

This formula is valid only for Compton up-scattering, i.e. energy transfer from the electrons to photons, and provides directly the inverse-Compton luminosities in the source frame and in the observer frame by applying the same transformations as for the synchrotron radiation above. 
We can neglect absorption of the inverse-Compton photons by electrons as long as frequencies of the emitted photons are high enough. However, at higher energies, the Compton photons may produce pairs by interacting with the synchrotron photons. This process can decrease the observed VHE radiation. The effect can be taken into account by using solution of the transfer equation for the spherical geometry (3) with the optical depth defined by:

$\tau_{\gamma \gamma}^{\prime}\left(\epsilon_{\mathrm{c}}^{\prime}\right)=0.2 \sigma_{T} \frac{1}{\epsilon_{\mathrm{c}}^{\prime}} n\left(1 / \epsilon_{\mathrm{c}}^{\prime}\right) R_{\mathrm{b}}$.

Such an expression is obtained from the integration of the number density of the synchrotron photons and the cross section for the pair production over the synchrotron frequencies (see e.g. Coppi \& Blandford 1990).

\subsection{Low-energy range: Inhomogeneous jet model}

Homogeneous models can not be used to explain the low frequency radio emission of Mrk 501 (see e.g. Pian et al. 1998) due to efficient self absorption. These models can not explain large (about 30 pc, e.g van Breugel \& Schilizzi 1986;) radio core observed at centimeter waves either. Therefore we admit more complicated inhomogeneous models to explain low frequency radiation of Mrk 501. Such kind of models (e.g. Marscher 1980; Konigl 1981; Ghisellini et al. 1985) usually assume a conical or paraboloid plus conical geometry of the emitting region, a power-law decrease of magnetic field and particle density along the jet as well as a constant value of Doppler factor.

To fit the radio up to the ultraviolet emission of Mrk 501 we adopt a simple version of the inhomogeneous jet model proposed by Ghisellini et al. (1985). We consider only a conical jet with geometry described by $r=a R$, where $r$ is the jet radius and $R$ the coordinate along the jet. The magnetic field intensity $B(R)$, the electron density $K(R)$ and the maximum electron energy $\gamma_{\max }$ are assumed to decrease along the jet as power-law functions:

$$
\begin{aligned}
B(R) & =B_{0}\left(R_{0} / R\right)^{m}, \\
K(R) & =K_{0}\left(R_{0} / R\right)^{n}, \\
\gamma_{\max }(R) & =\gamma_{\max }\left(R_{0}\right)\left(R_{0} / R\right)^{e} .
\end{aligned}
$$

The energy distribution of the relativistic electrons is assumed as well to be a power law function

$N(\gamma, R)=K(R) \gamma^{-s}$,

with the cut-off which decreases along the jet (see Eq. (16)). This assumption may be interpreted as a simple approximation of the situation where an initial power-law distribution of the electrons is only cooled by synchrotron radiation (see discussion in the Sect. 3.1). The inverseCompton cooling may be not efficient for the jet where electrons densities are smaller than in the blob.

The index $s$ will be directly estimated from the spectral index $\alpha_{0}$ of the optically thin radio synchrotron radiation $\alpha_{0}=(s-1) / 2$. The synchrotron luminosity in the jet frame is obtained by integrating the local volume emissivity $\varepsilon_{\mathrm{s}}^{*}\left[\mathrm{erg} \mathrm{s}^{-1} \mathrm{~cm}^{-3} \mathrm{~Hz}^{-1}\right.$ ] over the jet cone region

$L_{\mathrm{s}}^{*}\left(\nu_{\mathrm{s}}^{*}\right)=\int_{R_{1}\left(\nu_{\mathrm{s}}^{*}\right)}^{R_{2}\left(\nu_{\mathrm{s}}^{*}\right)} \pi a^{2} R^{2} \varepsilon_{\mathrm{s}}^{*} \mathrm{~d} R$

where the emissivity writes

$\varepsilon_{\mathrm{s}}^{*}=C_{1}\left(\alpha_{0}\right) K_{0} B_{0}^{1+\alpha_{0}} \nu_{\mathrm{s}}^{*-\alpha_{0}}\left(R_{0} / R\right)^{n+m\left(1+\alpha_{0}\right)}$,

where $C_{1}\left(\alpha_{0}\right)$ is given in Blumenthal \& Gould (1970). The integration boundaries $R_{1}$ and $R_{2}$

$R_{1}\left(\nu_{\mathrm{s}}^{*}\right)=R_{0}\left(\nu_{\mathrm{s}, \mathrm{m}}^{*}\left(R_{0}\right) / \nu_{\mathrm{s}}^{*}\right)^{1 / K_{\mathrm{m}}}$

$R_{2}\left(\nu_{\mathrm{s}}^{*}\right)=R_{0}\left(\nu_{\mathrm{s}, \max }^{*}\left(R_{0}\right) / \nu_{\mathrm{s}}^{*}\right)^{1 /(2 e+m)}$,

correspond respectively to the distance where the jet becomes opaque at frequency $\nu_{\mathrm{s}}^{*}$, and the maximum distance where the jet still is contributing to the frequency $\nu_{\mathrm{s}}^{*}$. The contribution from the opaque region is neglected. $\nu_{\mathrm{s}, \mathrm{m}}^{*}$ is the self-absorption frequency and $\nu_{\mathrm{s} \text {, max }}^{*}$ the maximum frequency produced by synchrotron emission at a given distance, $K_{\mathrm{m}}=2\left[n+m\left(1.5+\alpha_{0}\right)-1\right] /\left(5+2 \alpha_{0}\right)$. The observed flux density is then obtained after applying the Doppler boosting, where $\delta_{\mathrm{j}}$ is now the Doppler factor of the conical jet. The jet will of course emit a small high energy selfCompton radiation in the range of $10^{22} \mathrm{~Hz}$ to $10^{24} \mathrm{~Hz}$. However, the observational data presently available are far from providing any constraint on this inverse-Compton part, thus we do not further analyze it here.

\subsection{The external Compton scattering}

Several external photons can interact with the relativistic particles of the blob by inverse-Compton mechanism, namely the synchrotron radio emission of the conical jet, the direct radiation from the accretion disc, and the disc radiation scattered and reprocessed by clouds and intercloud medium. They can of course produce gamma rays in addition to those of the SSC scenario.

We mainly consider here the contribution to the Compton scattering due to the soft photons produced by the jet itself, which is a characteristic of our "blob-in-jet" scenario. To estimate it, we have to transform their radiation field into the blob frame. The energy of the jet photon in the blob frame is shifted on the average by one order of magnitude to the highest frequency $E_{\mathrm{j}}^{\prime} \sim \Gamma_{\mathrm{r}} E_{\mathrm{j}}^{*}$ where $\Gamma_{\mathrm{r}}=1 / \sqrt{\left(1-\left(v_{\mathrm{b}}^{*} / c\right)^{2}\right)}$ is the Lorentz factor and $v_{\mathrm{b}}^{*}$ is the velocity of the blob defined in the jet frame and given by

$v_{\mathrm{b}}^{*}=\frac{v_{\mathrm{b}}-v_{\mathrm{j}}}{1-\frac{v_{\mathrm{b}} v_{\mathrm{j}}}{c^{2}}}$

where $v_{\mathrm{b}}$ and $v_{\mathrm{j}}$ are velocities of the blob and jet in the observer frame respectively. Following the transformation given by Inoue \& Takahara (1996) we can write the jet synchrotron intensity as seen in the blob frame as:

$I_{\mathrm{j}}^{\prime}\left(\nu_{\mathrm{s}}^{\prime}\right)=\frac{1}{4 \pi} \int \mathrm{d} \Omega^{\prime} \delta^{3} I_{\mathrm{j}}^{*}\left(\nu_{\mathrm{s}}^{*}\right)=\Gamma_{\mathrm{r}} I_{\mathrm{j}}^{*}\left(\nu_{\mathrm{s}}^{*}\right)$, 
where the transformation of frequency is given by $\nu_{\mathrm{s}}^{*}=$ $\nu_{\mathrm{s}}^{\prime} / \Gamma_{\mathrm{r}}$ (see also Sikora et al. 1994).

In our calculations we assume that the blob is located inside the jet at the position $R_{x}$. The homogeneous blob can be understood as a perturbation or shock traveling down the jet. Its geometry in principle may be more complicated (e.g. similar to slice or cone) but for the sake of simplicity we keep spherical geometry as typical for homogeneous models. Similar models were adopted e.g. by Marscher \& Gear (1985), O'Dell (1988) and Hughes et al. $(1989 a, b)$ to explain the radio to infrared variability of blazars. For the high energy variability of blazars such a scenario was also tested e.g. by Celotti et al. (1991).

To estimate the synchrotron intensity in the jet at this position we take into account only the local contribution of the jet. Therefore we can approximate the intensity by solving the transfer equation for a part of jet with size equals to jet cross-section at blob position

$I_{\mathrm{j}}^{*}\left(\nu_{\mathrm{s}}^{*}, r_{x}\right)=\frac{1}{4 \pi} \frac{\varepsilon_{\mathrm{s}}^{*}\left(\nu_{\mathrm{s}}^{*}, r_{\mathrm{x}}\right)}{k\left(\nu_{\mathrm{s}}^{*}, r_{\mathrm{x}}\right)}\left(1-\exp \left(-r_{x} k\right)\right)$,

where $r_{x}=a R_{x}$.

To complete the picture, we will also test how the existence of relatively weak additional external radiation field can modify the inverse-Compton spectrum. We assume that the external radiation is isotropic and defined by a simple Planck function at temperature $T_{\mathrm{e}}$. External photons are blue-shifted in the blob frame. The maximum level of this radiation is set to be comparable with the blob radiation field which corresponds to the case of interest here, where it can have some influence without being the dominant mechanism (see Sect. 4.3).

\subsection{Absorption of the inverse-Compton radiation by interacting with intergalactic infrared medium}

Apart from the pair-production inside the source itself, there is also possibility for interaction between the VHE gamma-rays from the source and the Intergalactic Infrared Background (IIB). This can attenuate the high energy tail of the gamma ray spectra in blazars. To estimate influence of the effect we adopt the absorption coefficient of the infrared intergalactic radiation derived by Stecker \& De Jager (1998). They used a new empirical calculations of IIB made by Malkan \& Stecker (1998) from the observations of galaxies in infrared. The absorption coefficient was calculated for two values of the IR background which may be considered as a lower and upper limits. The absorption parameter is approximated by a 3rd order polynomial and is valid for redshift less than 0.3 . Spectra are calculated from the solution of the transfer equation with absorption only (e.g. Rybicki \& Lightman 1979).

\subsection{IR to UV range: The host galaxy}

The published optical magnitudes of Mrk 501 and of other nearby BL Lac objects have high dispersion. This results

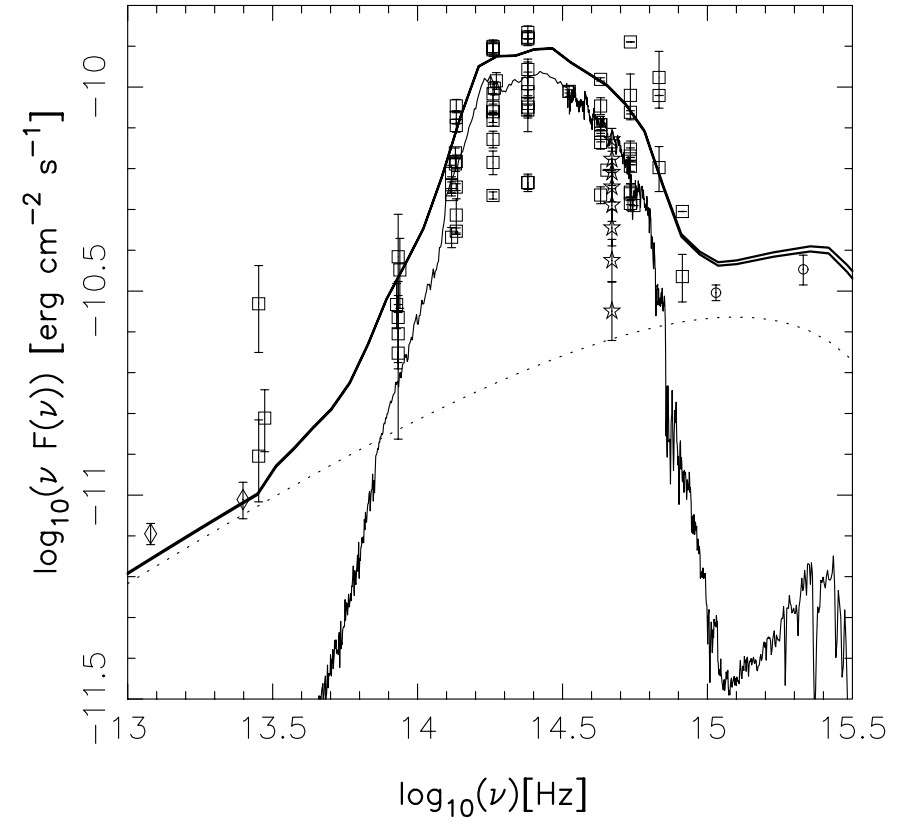

Fig. 1. The fit of the standard elliptical galaxy model (thin solid line), the synchrotron jet component (dotted line) for three different states and the sum of the host galaxy and the jet components (thick solid lines). Stars: estimated brightness of the host galaxy in the $R$-band obtained from the photometric decomposition (Nilsson et al. 1999) for different apertures. Diamonds: infrared data (Impey \& Gregorini 1993). Circles: UV data (Pian \& Treves 1993). Squares: data from NED database

from two effects. The main one is the undetermined contribution of the host galaxy, which depends on instrumental techniques, aperture or integration area used for photometric measurements, and seeing condition. This is well illustrated in papers where decomposition of images into the host galaxy and the nucleus has been performed and contribution of the host galaxy to the total flux carefully analyzed (e.g. Nilsson et al. 1999). The other effect responsible for dispersion in the optical fluxes is the intrinsic variability of the nucleus. However, it is very difficult to disentangle this real variability effect from spurious changes due to different host galaxy contribution in archival data. This is well illustrated in Fig. 1 where we collected all the data available from the NED data base and from published papers.

To fit the host galaxy contribution to the observed radiation of Mrk 501 we use a galaxy evolution model called PEGASE, developed by Fioc \& Rocca-Volmerange (1997). Assuming typical values of physical parameters for a big elliptical galaxy we generate synthetic spectrum for a galaxy of age equal to $16 \mathrm{~Gy}$. To calibrate the obtained spectrum we used the $R$-band photometric table obtained from a two-dimensional photometric decomposition of nucleus and host galaxy (Nilsson et al. 1999). Varying the mass of the galaxy only, the best fit is obtained for an elliptical of $3.910^{11}$ solar mass for $z=0.0377$ and $H_{0}=65 \mathrm{~km} \mathrm{~s}^{-1} \mathrm{Mpc}^{-1}$. 


\section{Constrains of the physical parameters for the homogeneous SSC model}

Within our SSC computation scheme, we have eight free independent parameters to describe the high-energy part of the spectra. Three parameters are related to the global properties of the emitting blob, namely $R_{\mathrm{b}}$ - the radius of the emitting blob, $B$ - the uniform magnetic field, and $\delta_{\mathrm{b}}$ - the Doppler factor. The five others parameters are related to the particle physics and describe the high-energy particle distribution, namely $K_{1}$ - the density factor, $\gamma_{\mathrm{b}}$ and $\gamma_{\text {cut }}$ - the Lorentz factors at the break and cut-off of the electron energy distribution, and $n_{1}, n_{2}$ the spectral indexes of the electron energy distribution at low and higher energies respectively (we further assume $\gamma_{\text {min }}=1$ which has no significant influence on the final high energy spectral shapes). The quality of the available high energy spectral data points allow us to directly deduce at least seven constraints. These are the values of the break and the peak frequencies in the synchrotron spectrum $\left(\nu_{\mathrm{s}_{\mathrm{b}}}, \nu_{\mathrm{s}_{\mathrm{p}}}\right)$, the peak frequency in the inverseCompton spectrum $\left(\nu_{\mathrm{c}_{\mathrm{p}}}\right)$, the three fluxes at these frequencies $\left(F_{\mathrm{s}}\left(\nu_{\mathrm{s}_{\mathrm{b}}}\right), F_{\mathrm{s}}\left(\nu_{\mathrm{s}_{\mathrm{p}}}\right), F_{\mathrm{c}}\left(\nu_{\mathrm{c}_{\mathrm{p}}}\right)\right)$, and the slope of the lowenergy synchrotron spectrum $\left(\alpha_{1}\right)$. Spectral data alone leave the system unconstrained, with still one degree of freedom. Further assumption or observational quantities, such as constraint on size of the emitting region, estimated from variability time scale, could in principle be used to get the unique answer if there were no uncertainties. In view of these uncertainties, it is more appropriate to derive relations between observed quantities and the physical parameters than to directly fix one single solution for the set of parameters. Therefore we extend in this section relations obtained by TMG, adding the cut-off in the electrons spectrum and providing more accurate approximation of the inverse-Compton spectrum.

\subsection{Particle energy distribution}

The power law distribution of particles energy with single break and cut-off appears to be the simplest approach to explain the observed high-energy spectra of Mrk 501 during active states of 1997 . The break is needed to reproduce spectral break around frequency $10^{17} \mathrm{~Hz}$ observed in X-rays during the medium state of April 7th, 1997, and to explain the smooth peak at the same band during the high state of April 16th, 1997. The cut-off is the simplest way to produce the synchrotron and inverse-Compton peaks and well reproduces the tail of the $\mathrm{TeV}$ radiation. This particle energy spectrum is somehow more complex than the ones used in previous basic SSC models of blazars (e.g. TMG) and introduces one additional free parameter. However, this complexity appeared necessary to us to obtain good fits of the new data, simultaneously collected in the X-rays and in the gamma-rays bands. The break splits the particle energy distribution into two parts with two different slopes $\left(n_{1}, n_{2}\right)$. These parameters can be constrained directly from the observations such that $\alpha_{i}=\left(n_{i}-1\right) / 2$ where $\alpha_{i}$ are the spectral indexes of the optically thin synchrotron source observed at the X-rays.

The local temporal evolution of the electron energy distribution in blazar's jets can be described in SSC model by the following partial differential equation

$$
\begin{aligned}
\frac{\partial N_{\mathrm{e}}(\gamma, t)}{\partial t}= & \frac{\partial}{\partial \gamma}\left[\left(C_{\mathrm{cool}}(\gamma, t)-C_{\mathrm{acc}}(\gamma, t)\right) N_{\mathrm{e}}(\gamma, t)\right] \\
& -\frac{N_{\mathrm{e}}(\gamma, t)}{t_{\mathrm{esc}}(\gamma, t)}+Q(\gamma, t)
\end{aligned}
$$

where $C_{\text {cool }}(\gamma, t)$ is the synchrotron self-Compton cooling rate, $C_{\text {acc }}(\gamma, t)$ the acceleration rate (for example in shock $), t_{\text {esc }}(\gamma, t)$ the escaping time and $Q(\gamma, t)$ a function describing injection of fresh electrons into the emitting region. Solutions of the equation may be used for calculation of the SSC spectra if the duration of physical processes like acceleration, cooling, injection and escaping is longer than the light crossing time $\left(R_{\mathrm{b}} / c\right)$. This assumption is true as long as the maximum energy of the electrons is not too high $\left(\gamma<10^{6}\right)$ and may be not true during flaring states. Additional calculations over the emitting region may then be necessary to obtain proper shape of the SSC spectra (e.g. Chiaberge \& Ghisellini 1999). However, we will not consider them here for sake of simplicity.

In a few cases, as shown for instance in Melrose (1980), the equation of electron energy distribution evolution (25) can be solved analytically and is useful to estimate the particle energy distribution shape. Neglecting acceleration, escaping, and injection and assuming the homogeneous blob we can expect almost uniform and constant cooling rate inside the source. Solution for such simplified equation can be written as (Kardashev 1962):

$N_{\mathrm{e}}(\gamma, t)= \begin{cases}K_{0} \gamma^{-a}\left(1-C_{\mathrm{c}} t \gamma\right)^{a-2} & \gamma<1 /\left(C_{\mathrm{c}} t\right) \\ 0 & \gamma>1 /\left(C_{\mathrm{c}} t\right),\end{cases}$

where

$N_{\mathrm{e}}(\gamma, 0)=K_{0} \gamma^{-a}, \quad C_{\mathrm{c}}=\frac{4}{3} \frac{\sigma_{\mathrm{T}}\left(U_{\mathrm{B}}+U_{\mathrm{r}}\right)}{3 m_{\mathrm{e}} c}=\frac{\gamma}{t_{\mathrm{cool}}}$,

and $t_{\text {cool }}$ is the cooling time which corresponds to the synchrotron and the inverse-Compton radiative losses in the Thompson regime. The solution (26) provides a power law function with a sharp cut-off at the electron energy $\gamma \sim 1 /\left(C_{\mathrm{c}} t\right)$. However, for more accurate calculation including the Klein-Nishina regime the inverse-Compton cooling rate should be a function of the electrons energy (see e.g. Chiaberge \& Ghisellini 1999 Eq. (17)).

More complete solutions of Eq. (25) were studied for example in detail by Kirk et al. (1998). Assuming injection of monochromatic electrons, constant escaping and particle acceleration by a shock, they obtained quite complicated results which can be reasonably well approximated by our assumption (1).

\subsection{Peak frequencies}

To obtain simple constraints on the physical parameters within our homogeneous synchrotron self-Compton model, 
we extend calculations made by TMG in the Klein-Nishina regime. The variable cut-off in the electrons energy distribution has been introduced to obtain a more accurate description.

A first relation between the magnetic field intensity and the Doppler factor is obtained from the values of the synchrotron and the inverse-Compton peaks frequencies. The Lorentz factor of the electrons emitting at the frequency $\nu_{\mathrm{s}_{(\mathrm{b} / \mathrm{p})}}$, corresponding to observable break or peak in the synchrotron spectrum, can be expressed as:

$\gamma_{\mathrm{b}_{(\mathrm{b} / \mathrm{cut})}}=\sqrt{\frac{(1+z) \nu_{\mathrm{s}_{(\mathrm{b} / \mathrm{p})}}}{C_{(\mathrm{b} / \mathrm{p})} B \delta_{\mathrm{b}}}}$,

where $C_{(\mathrm{b} / \mathrm{p})}$ is a constant with a rather long expression that we do not present here. For the break in the synchrotron spectrum $\left(\nu_{\mathrm{s}_{\mathrm{b}}}\right) C_{\mathrm{b}}=3.710^{6}$ (see also TMG). For the peak in the synchrotron spectrum $\left(\nu_{\mathrm{s}_{\mathrm{p}}}\right)$ which is related to the cut-off in the electrons spectrum $\left(\gamma_{\text {cut }}\right)$ value of $C_{\mathrm{p}}$ is given by approximation:

$$
\begin{aligned}
C_{\mathrm{p}}\left(n_{2}\right) \sim & -3.2510^{5} n_{2}^{3}+1.6710^{6} n_{2}^{2} \\
& -3.6210^{6} n_{2}+4.6510^{6},
\end{aligned}
$$

obtained from numerical calculations. This approximation is valid for $n_{2}=1.5 \div 2.99$. The relation between position of the inverse-Compton peak, the Doppler factor value and the $\gamma_{\text {cut }}$ is derived in the Appendix B. Unfortunately Eq. (B.3) cannot be solved analytically, but it is easy to solve it numerically using for example the bisection method. Substituting $\gamma_{\text {cut }}$ into Eq. (27), we then deduce a first relation between the magnetic field intensity and the Doppler factor value, which will be important in Sect. 4.1 to narrow the space of allowed parameters.

\subsection{Peak luminosities}

Another relation between the magnetic field intensity and the Doppler factor can be calculated from:

$\frac{F_{\mathrm{c}}}{F_{\mathrm{s}}}=\frac{U_{\mathrm{rad}_{\mathrm{av}}}^{\prime}}{U_{B}^{\prime}}$,

where $F_{\mathrm{S}}$ and $F_{\mathrm{c}}$ are the total emitted synchrotron and inverse-Compton fluxes, $U_{B}^{\prime}=B^{2} /(8 \pi)$ is the magnetic field energy density, and $U_{\mathrm{rad}_{\mathrm{av}}}^{\prime}$ is the available synchrotron radiation energy density given by:

$U_{\mathrm{rad}_{\mathrm{av}}}^{\prime}=\int_{\nu_{\mathrm{sin}}^{\prime}}^{3 m_{\mathrm{e}} c^{2} / 4 h \gamma_{\mathrm{b}}} u_{\mathrm{r}}\left(\nu_{\mathrm{s}}^{\prime}\right) \mathrm{d} \nu_{\mathrm{s}}^{\prime}$,

where

$u_{\mathrm{r}}\left(\nu_{\mathrm{s}}^{\prime}\right)=G \nu_{\mathrm{s}}^{\prime-\alpha_{1}}$.

Neglecting a contribution from the lower integral boundary this reduces to:

$U_{\mathrm{rad}_{\mathrm{av}}}^{\prime}=\frac{G}{1-\alpha_{1}}\left(\frac{3 m_{\mathrm{e}} c^{2}}{4 h \gamma_{\mathrm{b}}}\right)^{1-\alpha_{1}}$, where $G$ is expressed in terms of the Doppler factor, the emitting volume radius $R_{\mathrm{b}}$ and of other observable parameters:

$$
G=\frac{9}{4} \frac{F_{\mathrm{s}}\left(\nu_{\mathrm{s}_{\mathrm{b}}}\right) d_{\mathrm{l}}^{2}}{c R_{\mathrm{b}}^{2} \delta_{\mathrm{b}}^{3}(1+z)\left(\frac{\nu_{\mathrm{s}}(1+z)}{\delta_{\mathrm{b}}}\right)^{-\alpha_{1}}} .
$$

Approximating the electrons spectrum by the broken power-law function with the sharp cut-off we can express the total synchrotron flux as:

$$
\begin{aligned}
F_{\mathrm{s}}= & \nu_{\mathrm{s}_{\mathrm{b}}} F_{\mathrm{s}}\left(\nu_{\mathrm{s}_{\mathrm{b}}}\right)\left(\frac{1}{1-\alpha_{1}}+\frac{1}{\alpha_{2}-1}\right) \\
& +\nu_{\mathrm{s}_{\mathrm{p}}} F_{\mathrm{s}}\left(\nu_{\mathrm{s}_{\mathrm{p}}}\right)\left(\frac{1}{1-\alpha_{2}}\right) .
\end{aligned}
$$

Having quite good approximation of the synchrotron part of the spectrum we derive also more accurate approximation for the inverse-Compton part of the spectrum described in the Appendix C (Eq. (C.1)).

An additional independent constraint on the radius of the emitting region can be estimated from the observed variability time scales $t_{\mathrm{var}}, R_{\mathrm{b}} \leq c t_{\mathrm{var}} \delta_{\mathrm{b}} /(1+z)$. We therefore reach a second relation between $B$ and $\delta_{\mathrm{b}}$, which depends only on directly observable quantities, whose importance is illustrated below.

\section{Application to Mrk 501}

We focus here on the two specific high states shown by Mrk 501 in April 1997, during a quite long-term increasing activity visible in the X-ray light curve obtained from XTE/ASM (e.g. Ghisellini 1998). The available data are very good for testing the emission mechanisms from X-rays to VHE because observations were made simultaneously in both X-rays by BeppoSAX and gamma-rays by CAT.

\subsection{The spectral fits of high energy emission}

As described in Sect. 3, we deduce constraints on the Doppler factor and the magnetic field intensity from observable quantities. These quantities have been estimated from the observed spectra shown in Fig. 2 and are given in Table 1.

The synchrotron spectral breaks appear more clearly in the spectrum at the medium state of April 7th, 1997, which we analyze first. Taking into account uncertainty in the position of the inverse-Compton peak and assuming $R_{\mathrm{b}}$ equal to $5.010^{15} \mathrm{~cm}$ for this medium state, we obtain the allowed parameter space for $B$ and $\delta_{\mathrm{b}}$. We then start a series of spectrum fits with these initial constraints and finally get the best fit for a value of the Doppler factor equals to 14 , a magnetic field intensity equals to $0.2 \mathrm{G}$, and a radius equals to $4.210^{15} \mathrm{~cm}$. Such a radius corresponds to a variability time scale of about 3 hours which is quite realistic in view of available gamma-rays light curves (Quinn et al. 1999). 

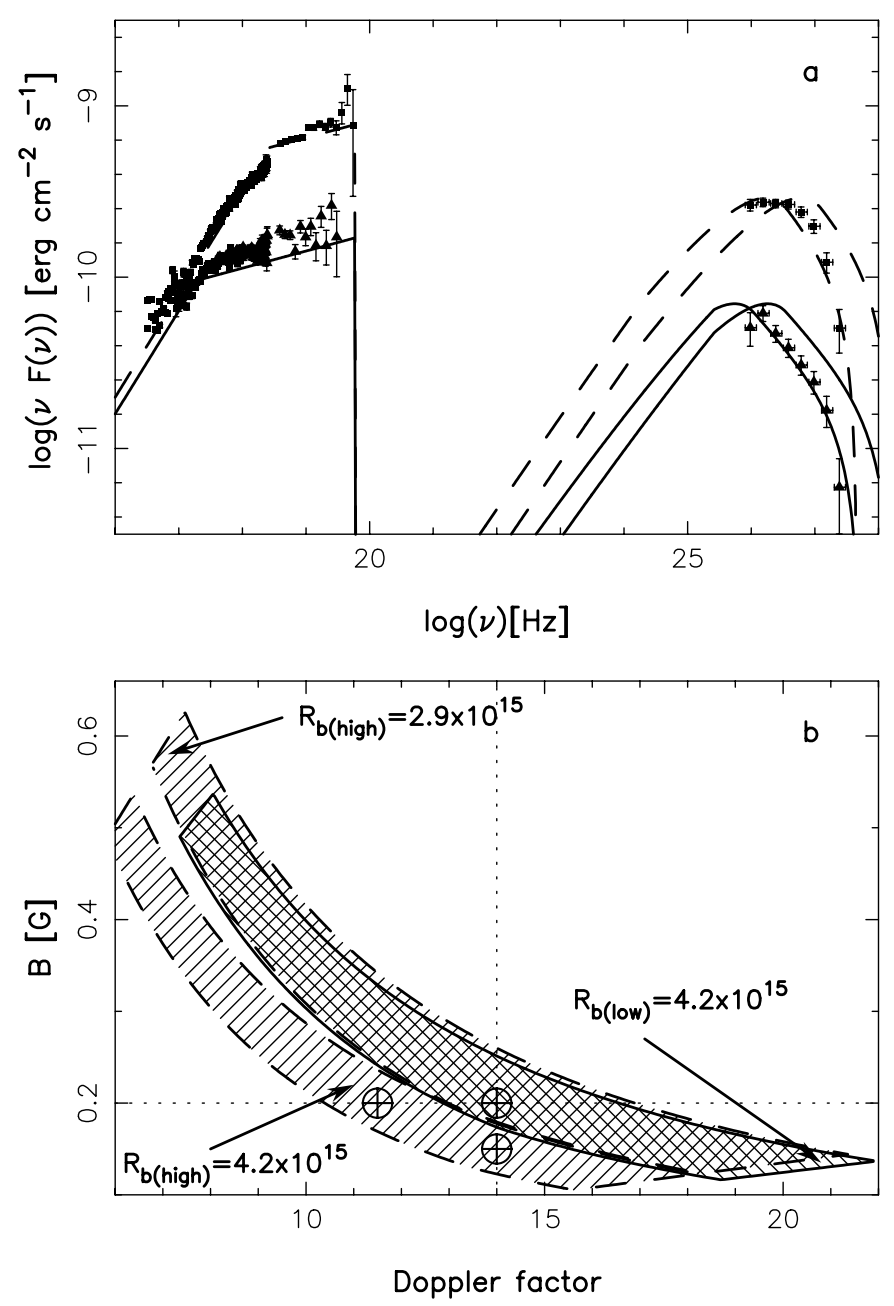

Fig. 2. Constraints on the Doppler factor and the magnetic field values. The upper picture a) shows assumed spectral shapes used for calculations (see for quantities in Table 1). The lower picture b) shows allowed space of parameters. For the medium state allowed parameter space is limited by full lines. For the high state (dashed boundaries) we calculate two allowed spaces for two different values of the blob radius $R_{\mathrm{b}}$. The three different sets of parameters were obtained independently by direct fits of the spectra, illustrated in Fig. 3. Here they are indicated by three crossed circles

In the next steep we try to fit the high energy state of April, 16th, 1997, first by keeping the values of the global physical parameters $\left(B, R_{\mathrm{b}}, \delta_{\mathrm{b}}\right)$ equal to those of the medium state, allowing only a small shift towards higher energies of $\gamma_{\mathrm{b}}$, the break in the electron energy spectrum, and an increase of the particle density. This would describe an evolution of the medium state into the high state just by injection or acceleration of high energy electrons. Such a scenario provides a very good fit for the synchrotron spectrum and a quite good shape of the inverse-Compton spectral peak but the level of this spectrum always remains a little bit too low. This directly suggests that the efficiency of the inverse-Compton radiation was higher during the high state of April 16th than during the previous one of April 7th.
Table 1. The observed quantities of the high energy emission of Mrk 501 used for estimating the allowed parameter space for the medium and the high states

\begin{tabular}{llll}
\hline & medium state & high state & \\
\hline$\alpha_{1}\left(n_{1}\right)$ & $0.40(1.80)$ & $0.40(1.80)$ & \\
$\alpha_{2}\left(n_{2}\right)$ & $0.90(2.80)$ & $0.90(2.80)$ & \\
$\nu_{\mathrm{s}_{\mathrm{b}}}$ & $2.0010^{17}$ & $2.7010^{18}$ & $\mathrm{~Hz}$ \\
$\nu_{\mathrm{s}_{\mathrm{p}}}$ & $6.0010^{19}$ & $6.0010^{19}$ & $\mathrm{~Hz}$ \\
$F_{\mathrm{s}}\left(\nu_{\mathrm{s}_{\mathrm{b}}}\right)$ & $4.8110^{-28}$ & $2.1210^{-28}$ & $\mathrm{erg} \mathrm{cm}^{-2}$ \\
$F_{\mathrm{s}}\left(\nu_{\mathrm{S}_{\mathrm{p}}}\right)$ & $2.8310^{-30}$ & $1.3010^{-29}$ & $\mathrm{erg} \mathrm{cm}^{-2}$ \\
$\nu_{\mathrm{c}_{\mathrm{p}, \min }}$ & $0.5510^{26}$ & $1.7010^{26}$ & $\mathrm{~Hz}$ \\
$\nu_{\mathrm{c}_{\mathrm{p}, \max }}$ & $1.8010^{26}$ & $6.0010^{26}$ & $\mathrm{~Hz}$ \\
$F_{\mathrm{c}}\left(\nu_{\mathrm{c}_{\mathrm{p}, \min }}\right)$ & $1.2710^{-36}$ & $1.7110^{-36}$ & $\mathrm{erg} \mathrm{cm}^{-2}$ \\
$F_{\mathrm{c}}\left(\nu_{\mathrm{c}_{\mathrm{p}, \max }}\right)$ & $3.8910^{-37}$ & $4.8310^{-37}$ & $\mathrm{erg} \mathrm{cm}^{-2}$ \\
\hline
\end{tabular}

We therefore estimate the allowed $B-\delta_{\mathrm{b}}$ parameter space for the high state also, and find it shifted towards lower values of the magnetic field and Doppler factor relatively to the medium state, assuming the same radius $R_{\mathrm{b}}$ (see Fig. 2). However, the allowed $B-\delta_{\mathrm{b}}$ parameter space for the two states are found very similar when assuming a slightly smaller radius for the high state, namely $R_{\mathrm{b}}=2.910^{15} \mathrm{~cm}$, of which the size corresponds to a variability time scale of about two hours. With this decrease of $R_{\mathrm{b}}$ and the previously mentioned changes of $\gamma_{\mathrm{b}}$ and $K_{1}$ we finally obtain a first very good fit (labelled "h 1" in Table 2) for the spectral distribution observed on April 16th.

There are in fact two ways to increase the efficiency of the inverse-Compton scattering. The first method is to increase the photon density. However, for SSC models the density is directly calculated for a given magnetic field and particle energy spectrum, and there is no possibility to modify this parameter. The method can nevertheless be useful for models which also take into account external radiation fields, discussed in the next section. In SSC models, a second possibility to increase efficiency of the inverse-Compton radiation is to assume higher particle density. Increasing particle density allows easily to reach the required level of the inverse-Compton spectrum in high state since its peak is proportional to $K_{1}^{2}$. A direct consequence is also the corresponding growth of the synchrotron flux which is this time proportional to $K_{1}$. To compensate this effect and keep the same satisfactory level of the synchrotron spectrum, it is necessary to decrease simultaneously one of the global parameters, $R_{\mathrm{b}}$ (as found above), $B$, or $\delta_{\mathrm{b}}$. Indeed we obtain two other very good fits of the high state spectrum (labelled "h 2 " and "h 3 " in Table 2) by slightly decreasing values of the magnetic field or the Doppler factor alone, to compensate the effect of increasing $K_{1}$. Only one fit is shown in Fig. 3 (the one corresponding to "h 1 " with a decrease of the radius) as the three satisfactory fits are very similar. Such variation 
Table 2. The physical parameters of our synchrotron selfCompton model derived from interactive fits of the spectra for the medium (m) and high (h) states. Three different parameter sets are possible. For the SSC model we present all possibilities, for the SSC model and absorption in IIB only one possibility. However other analogous to the pure SSC calculations are also possible

\begin{tabular}{llllllll}
\hline & & SSC & & \multicolumn{3}{c}{ SSC + abs. } \\
& & $\mathrm{m}$ & $\mathrm{h} \mathrm{1}$ & $\mathrm{h} \mathrm{2}$ & $\mathrm{h} \mathrm{3}$ & $\mathrm{m}$ & $\mathrm{h}$ \\
\hline$R_{\mathrm{b}}$ & $\left(10^{15} \mathrm{~cm}\right)$ & 4.2 & 2.9 & 4.2 & 4.2 & 4.2 & 2.9 \\
$B$ & $(\mathrm{G})$ & 0.2 & 0.2 & 0.15 & 0.2 & 0.15 & 0.15 \\
$\delta_{\mathrm{b}}$ & & 14 & 14 & 14 & 11.5 & 14 & 14 \\
$K_{1}$ & $\left(10^{3} \mathrm{~cm}^{-3}\right)$ & 1.0 & 2.7 & 1.6 & 1.9 & 1.0 & 3.4 \\
& & & & & & & \\
$\gamma_{\mathrm{b}}$ & $\left(10^{5}\right)$ & 1.1 & 6.0 & 6.0 & 6.0 & 2.2 & 8.5 \\
$\gamma_{\text {cut }}$ & $\left(10^{6}\right)$ & 3.0 & 3.0 & 3.0 & 3.0 & 4.0 & 4.0 \\
$n_{1}$ & & & & & & & \\
$n_{2}$ & & 1.8 & 1.8 & 1.8 & 1.8 & 1.8 & 1.8 \\
\hline
\end{tabular}

of the global parameters are in good agreement with the constraints derived previously and illustrated in Fig. 2.

After these preliminary fits we introduce to our calculation absorption due the pair-production inside source and in the intergalactic infrared medium. We find that the first of these absorption processes is almost negligible but the second can introduce significant changes to the spectral shapes. This results is in agreement with the results obtained by Bednarek \& Protheroe (1999). From detailed fits we also find that strong absorption can modify drastically the shape of the VHE emission and are unable to obtain good spectral fits using higher absorption conditions from those proposed by Stecker \& De Jager (1998). Finally assuming relatively weak absorption by IIB we get very good fits with only small modification of the physical parameters which we first obtain for the pure SSC model. Similar results were derived recently by Tavecchio \& Maraschi (2000).

Good fits of the two states are therefore obtained without varying three of our parameters $\left(\gamma_{\text {cut }}, n_{1}\right.$ and $\left.n_{2}\right)$. To reproduce the two medium and high states, variations of at least three other parameters appear necessary $-\gamma_{\mathrm{b}}$ and $K_{1}$, plus one of the global parameters of the emitting blob, $R_{\mathrm{b}}, B$, or $\delta_{\mathrm{b}}$.

In Table 3 we compare a few results of different authors. The constraints for the physical parameters deduced from our accurate approach and the values obtained from the detailed fits remain in good agreement with other recent calculations. The only exception is the preliminary calculations made by TMG which concludes to different values. However, they were not consistent with the third $\delta-B$ relation obtained by TMG from the equilibrium between the escaping and cooling times for electrons with energy $\gamma_{\mathrm{b}}$, which we do not discuss here. This problem
Table 3. Comparison of the physical parameters obtained from fits of the SSC models to high energy states observed in Mrk 501

\begin{tabular}{lllll}
\hline Author & $B[\mathrm{G}]$ & $\delta$ & $t_{\text {var }}[\mathrm{h}]$ & IIB abs. \\
\hline TMG & $1-32$ & $2-7$ & 3.3 & no \\
Tavecchio et al. (2000) & 0.02 & 10 & 16 & no \\
& 0.005 & 10 & 32 & low \\
this paper & 0.2 & 14 & 1.9 & no \\
& 0.15 & 14 & 1.9 & low \\
Kataoka et al. (1999) & 0.2 & 15 & 2.8 & no \\
Guy et al. (2000) & 0.05 & 15 & 10 & high \\
Bednarek et al. (1999) & 0.1 & 11.5 & 2.5 & low \\
& 0.07 & 11.2 & 2.5 & high \\
\hline
\end{tabular}

can be solved as shown by Guy et al. (2000), assuming relatively strong absorption by IIB. Anyway, more recent work by Tavecchio \& Maraschi (2000) also conclude to similar values of physical parameters.

\subsection{The radio emission}

Several new parameters appear in the model of inhomogeneous conical jet, which we adopt to describe the radiation of Mrk 501 from the radio up to the ultraviolet ranges. They are related to the geometry of the jet, to the particle energy distribution and to the magnetic field. Spectral data alone are clearly insufficient to constrain all these parameters and we have to consider other astrophysical properties as well.

As a first step, we put constraints on geometry of the cone. Typical inner radius of the jet $-r_{0}$, is likely linked to the Schwarzchild radius of the central black hole. This can be obtained from an estimate of the black hole mass, which is related to the magnitude of the bulge of host galaxy. The magnitude of the Mrk 501 galaxy in the blue band is 14.5 (e.g. Mufson et al. 1984; Xie et al. 1999), what corresponds to an absolute magnitude of -21.5 . From the relation between the absolute magnitude of host galaxies and the mass of their central black holes, derived by Kormendy \& Richstone (1995), we obtain a mass of $10^{9}$ solar mass for the central black hole in Mrk 501. This is very close to the values usually admitted in the case of the nearby radiogalaxy $\mathrm{M} 87$, in fact which could be a misdirected BL Lac object. The most inner stable circular orbit around a Schwarzschild black hole is located at a distance three times bigger than the Schwarzschild radius, thus here about $10^{15} \mathrm{~cm}$. Typical width of the jet should be then a few times larger. We therefore assume the inner radius of the conical jet equal to $10^{16} \mathrm{~cm}$. Basic constraints on the outer parts of the jet can be estimated from radio maps of the source obtained at centimeter wavelengths. 


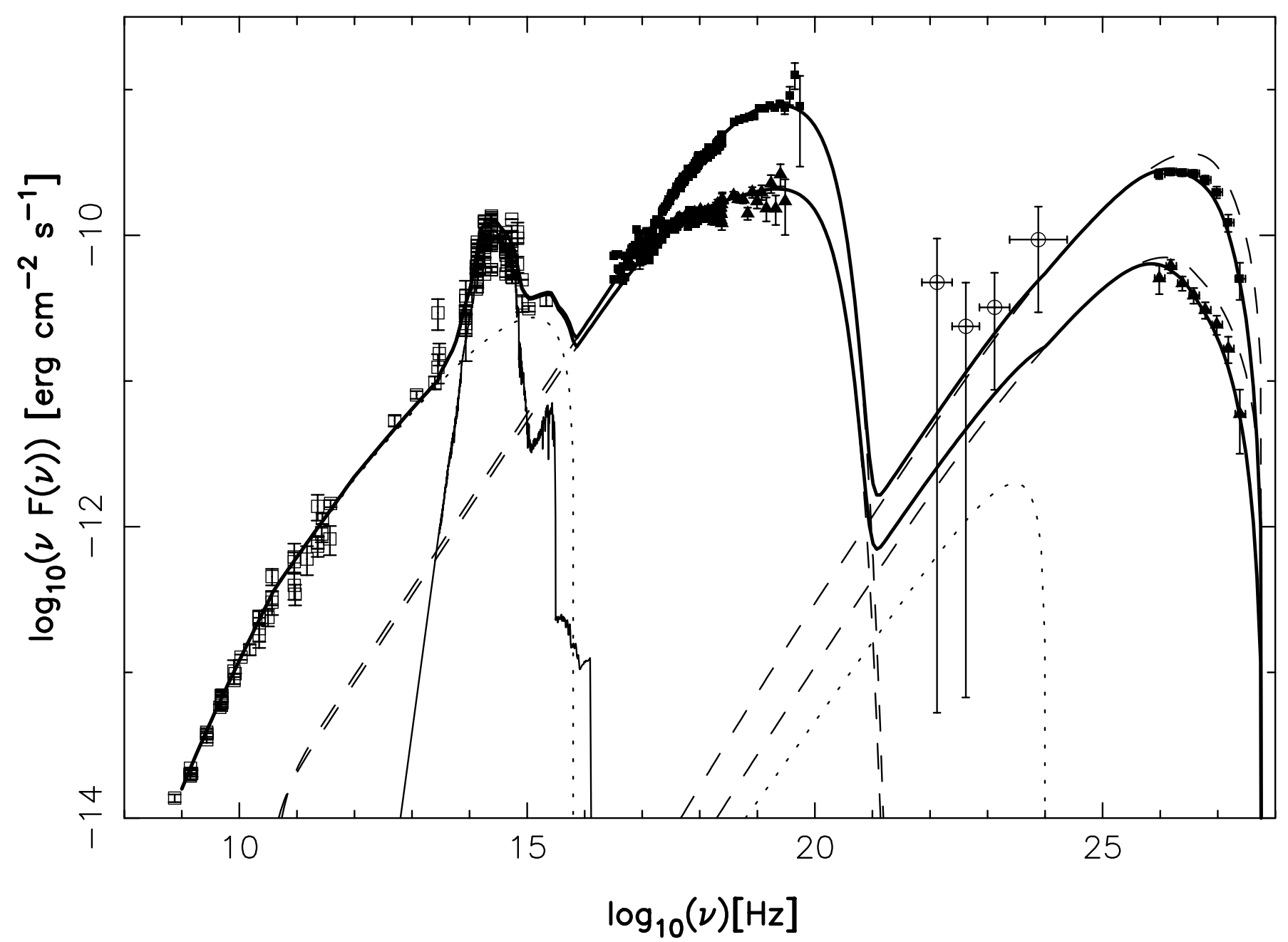

Fig. 3. Radio to TeV energy distribution of Mrk 501. BeppoSAX (Pian et al. 1998) and simultaneous CAT (Djannati-Atai et al. 1999) data from 7 (triangles) and 16 (boxes) April 1997. The circles show the first detection of Mrk 501 by EGRET from March 1996 (Kataoka et al. 1999). Non-simultaneous data collected from literature (Impey \& Gregorini 1993; Gear 1993; Gear et al. 1994; Steppe et al. 1988; Wiren et al. 1992; Lawrence et al. 1991; Pian \& Treves 1993) and NED database (open boxes). The thick solid lines indicate fits of the multiwavelength emission of the source for two different states of activity. The dashed lines correspond to the SSC emission without absorption by the IIB. The dotted lines indicate radiation of the conical jet and thin solid lines shows contribution of the host galaxy emission

The conical jet model can explain only the radiation emitted by the central core. The core can be seen in VLBI maps and has extension of $30 \mathrm{pc}$ at $6 \& 5 \mathrm{~cm}$ (e.g. van Breugel \& Schilizzi 1986; Conway \& Wrobel 1995). We assume a half opening angle of the jet $\psi$ equal to two degrees and a jet length equal to $150 \mathrm{pc}$. The corresponding value of the outer radius of the cone is then $r_{\max } \sim 5$ pc. For a realistic angle of two degrees between the main axis of the jet and the line of sight, the apparent size of the jet in the plane of sky should be of about $10 \mathrm{pc}$, which remains in good agreement with the structure seen on available radio maps.

The observed spectral index from the lowest radio frequencies up to the optical range is about 0.5 , which imposes the value of the index of the particle energy spectrum, $s \simeq 2$. The exact spectral shape computed from the conical model depends also on the variation of the particle distribution and of the magnetic field intensity along the jet. For the basic solution of the evolution described in Eq. (25) with constant cooling, no acceleration, no escaping and no injection $\gamma_{\max }$ evolves as $R^{-1}$ (Eq. (26)) for a population of particles propagating at a constant speed along the jet, and the index $e$ should be equal to 1. However, it is impossible to generate a good shape for the spectrum within such a scenario which appears too simple. Therefore we allow variation of $e, m$ and $n$, three parameters still unconstrained defined in Eqs. (14)-(16). The best fit for the observed spectral shape is obtained for the following values $e=0.15, m=0.9$ and $n=1.8$. Note that basic solutions which conserve magnetic flux and particle number along the jet $(m=n \simeq 2)$ do not produce good spectral patterns. Internal physics of the jet has to be more complex.

Finally, we have to fix-up the level of the emitted energy, which depends only on magnetic field intensity, density of the particles and Doppler factor value. For the radio 
synchrotron part of the spectrum good fits can be obtained for relatively small magnetic field and high particle density and for the opposite case as well. However, if the particle density is too large the efficiency of the inverse-Compton emission in the radio jet becomes too high and then starts to contribute significantly to the gamma-ray flux of the source. This should be avoided in the present scenario of Mrk 501, where the high energy emission is explained by the SSC emission of a simple homogeneous blob, which fulfills the fundamental constraint based on argument of the observed variability time scales of about few hours (Quinn et al. 1999). Thus we impose a particle density small enough to keep the inverse-Compton radiation from the radio jet itself relatively small as compared with the gamma-ray emission of the blob. On the other side, we constrain the magnetic field in the jet to have intensity similar to the value inside the blob at the location of the blob (0.15 G, derived from Sect. 4.1). This may overestimate the jet magnetic field if magnetic field is compressed with plasma (e.g. by a shock) and intensified inside the blob, but there is no observational data to fix this ratio and we select the simplest view. From the above assumptions we derive a magnetic field of about $0.35 \mathrm{G}$ at the base of the jet. Finally to obtain the observed level of synchrotron radiation from the jet we have to set the value of the jet Doppler factor $\left(\delta_{\mathrm{j}}\right)$ equal to seven. This quite large value is dictated also by the fact that we want here to avoid contributing of soft photons from the jet into inverse-Compton scattering in the blob. This additional scattering can complicate significantly the generation of the VHE spectrum, and modify simple constrains for the physical parameters, derived in Sect. 4. Values of the physical parameters we adopt for the conical radio jet model are given in Table 4. More detailed analysis of the inverseCompton scattering of the external photons by the blob particles is discussed in the next section.

\subsection{Inverse-Compton scattering of external photons}

As shown in Sect. 2.3, the two external fields of soft photons can interact with the blob particles and produce gamma-rays by inverse-Compton scattering, in addition to the synchrotron photons generated by the blob itself. The SSC models can clearly very well explain VHE radiation of blazars. However, EIC effect can still play some role since for instance in sources which do not show TeV activity, it seems to provide better solutions to understand the observed spectra (e.g. Inoue \& Takahara 1996). Therefore we treat here the EIC scattering as a complementary effect and check how the additional external radiation field can modify inverse-Compton spectra generated within the SSC scenario.

Firstly we explore the influence of the radio jet radiation. We decrease value of the jet Doppler factor $\left(\delta_{\mathrm{j}}=7 \rightarrow\right.$ $2.0)$ to make the interaction more efficient by increasing Doppler boosting effect between blob and jet. We increase volume $\left(r_{0}=10^{16} \rightarrow 4.010^{16} \mathrm{~cm}\right)$ and particle density
Table 4. Physical parameters for the "blob-in-jet" model. The first group of parameters is estimated directly from various data within the adopted "blob-in-jet" scenario (see text). The second group is derived. Parameters labelled by ${ }^{*}$ describe the jet properties at the position of the blob inside the jet

\begin{tabular}{|c|c|c|c|c|}
\hline estimated & & & & \\
\hline$\alpha_{0}$ & 0.45 & & & \\
\hline$m$ & 0.9 & & & \\
\hline$n$ & 1.8 & & & \\
\hline$e$ & 0.15 & & & \\
\hline$B_{0}$ & 0.35 & $\mathrm{G}$ & & \\
\hline$K_{0}$ & $1.010^{2}$ & $\mathrm{~cm}^{-3}$ & & \\
\hline$\psi$ & 2.0 & $\circ$ & & \\
\hline$\theta$ & 2.0 & $\circ$ & & \\
\hline$r_{0}$ & $1.010^{16}$ & $\mathrm{~cm}$ & $\left(3.210^{-3} \mathrm{pc}\right)$ & \\
\hline$r_{\max }$ & $1.610^{19}$ & $\mathrm{~cm}$ & $(5.2 \mathrm{pc})$ & \\
\hline$\nu_{\max }\left(R_{0}\right)$ & $1.010^{15}$ & $\mathrm{~Hz}$ & & \\
\hline$\delta_{\mathrm{j}}$ & 7.0 & & & \\
\hline \multicolumn{5}{|l|}{ derived } \\
\hline$R_{0}$ & $2.910^{17}$ & $\mathrm{~cm}$ & $\left(9.310^{-2} \mathrm{pc}\right)$ & \\
\hline$r_{\max }$ & $1.610^{19}$ & $\mathrm{~cm}$ & $(5.2 \mathrm{pc})$ & \\
\hline$R_{\max }$ & $4.610^{20}$ & $\mathrm{~cm}$ & $\left(1.510^{2} \mathrm{pc}\right)$ & \\
\hline$r_{x}$ & $2.610^{16}$ & $\mathrm{~cm}$ & $\left(8.410^{-3} \mathrm{pc}\right)$ & $*$ \\
\hline$R_{x}$ & $7.410^{17}$ & $\mathrm{~cm}$ & $\left(2.410^{-1} \mathrm{pc}\right)$ & $*$ \\
\hline$B_{x}$ & 0.15 & $\mathrm{G}$ & & $*$ \\
\hline$K_{x}$ & $3.310^{1}$ & $\mathrm{~cm}^{-3}$ & & $*$ \\
\hline
\end{tabular}

$\left(K_{0}=10^{2} \rightarrow 1.510^{2} \mathrm{~cm}^{-3}\right)$ of the jet to obtain higher densities of the radiation field itself. The additional radiation field increases efficiency of the inverse-Compton radiation thus we have to modify some of the blob parameters to keep the observed level of radiation $\left(K_{1}=3.410^{3} \rightarrow\right.$ $\left.410^{2} \mathrm{~cm}^{-3}, R_{\mathrm{b}}=2.910^{15} \rightarrow 5.810^{15} \mathrm{~cm}\right)$. This calculation shows that the inverse-Compton scattering with the jet photons can provide also very good fits of the VHE radiation of blazars. The spectral shapes of such radiation are very similar to the spectral shapes generated with SSC models (see Fig. 4; see also Ghisellini 1999). The "blob-injet" scenario with EIC from the radio jet itself can provide an interesting alternative to pure SSC models.

The second source of soft photons is the medium surrounding the jet. If the blob is located at a distance much larger than the outer radius of the accretion disk then photons coming directly from it are strongly red-shifted in the frame of the relativistically moving blob and are not good candidates to produce high energy radiation by inverse-Compton scattering. In opposite case where distance from the disc is comparable or smaller than the size of the accretion disc, thermal photons may be strongly blue-shifted in the blob frame and be quite important for the inverse-Compton scattering. On the other side, a part of disk radiation is likely to be reprocessed or scattered by 

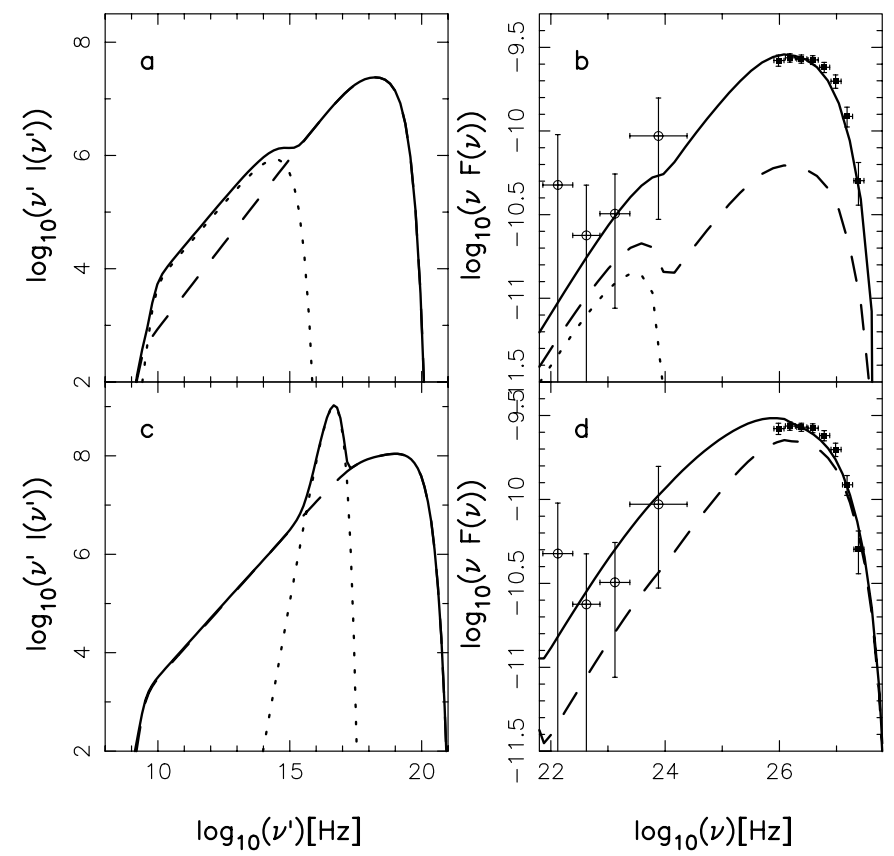

Fig. 4. Examples of the possible influence of two different external radiation fields, in addition to the basic SSC radiation of the homogeneous blob. Upper pictures $\mathbf{a}, \mathbf{b}$ ) illustrate modifications made by the synchrotron radiation field from the jet. Lower pictures c, d) show changes due to external thermal radiation. Pictures on the left $\mathbf{a}, \mathbf{c})$ present the synchrotron intensity of the blob itself (dashed lines), external intensities transformed into the blob frame (dotted lines) and the total intensities (solid lines). Pictures on the right $\mathbf{b}, \mathbf{d}$ ) show pure inverse-Compton spectra of the blob (dashed lines) and the modification of this spectra due to the different external radiation field (solid lines). The dotted line on the picture $\mathbf{b}$ ) shows the inverse-Compton radiation of the inhomogeneous jet

clouds and intercloud medium surrounding the blob, filling this space by a radiation field almost isotropic in the central engine frame. This radiation is highly blueshifted in the blob frame and can be a good target to produce high energy photons (see e.g Sikora et al. 1994; Sikora et al. 1996).

The intensities of the disc radiation and of the reprocessed radiation at the blob position, depends on distance to the accretion disc, physical conditions around the central engine, clouds and intercloud region properties. Estimate of this external radiation is possible only in few BL Lac objects (e.g. Bottcher \& Bloom 2000) and is difficult in the case of Mrk 501 in view of the present available optical data. Anyway, recent observations made by EGRET (Kataoka et al. 1999) show no significant influence of the thermal external radiation which should induce inverse-Compton emission at frequencies from $10^{21}$ to $10^{25} \mathrm{~Hz}$. Thus the effect of this EIC process is not expected to be dominant and we only test how relatively weak thermal radiation modifies SSC spectra.

To analyze the influence of the radiation field due to the ambient external medium, we assume maximum intensity $\left(\nu_{\max }^{\prime} I_{\max }^{\prime}=10^{9} \mathrm{erg} \mathrm{s}^{-1} \mathrm{~cm}^{-2}\right.$ sterad $\left.^{-1}\right)$ in the blob frame to be comparable with the blob synchrotron intensity. We describe it as a black body radiation at temperature $T_{\mathrm{e}}=5.010^{4} \mathrm{~K}$. This assumption is somewhat arbitrary. However, we can constrain upper limit for the central nucleus (accretion disc and medium surrounding it) flux directly from the spectrum energy distribution shown in Fig. 3, namely $\nu_{\max } F_{\text {nuc }}\left(\nu_{\max }\right) \leq 10^{-11} \mathrm{erg} \mathrm{s}^{-1} \mathrm{~cm}^{-2}$ in the UV range. The upper limit for the luminosity is then given by $\nu_{\max } L_{\text {nuc }}\left(\nu_{\max }\right) \leq 2.810^{43} \mathrm{erg} \mathrm{s}^{-1}$ and the total luminosity of the nucleus should be less than $2.7510^{43} \mathrm{erg} \mathrm{s}^{-1}$. Transforming the intensity assumed in the blob frame into the stationary frame $\left(\nu_{\max } I_{\max }=\right.$ $\nu_{\max }^{\prime} I_{\max }^{\prime} / \Gamma_{\mathrm{b}}^{2}$ see Eq. (23)) and using Eq. (5) we can estimate distance from the central engine to the blob position. For our upper limit of the nucleus luminosity we can obtain the assumed level of the intensity at the distance $210^{17} \mathrm{~cm}$. Indeed this approach provides simple way to estimate the distance of the blob from the nucleus. If luminosity of the nucleus is less or if radiation is significantly absorbed then contribution of this emission at such a distance should be negligible. Finally to obtain good spectral fits we have to modify also some of the blob parameters like in the previous case $\left(K_{1}=3.410^{3} \rightarrow\right.$ $2.610^{3} \mathrm{~cm}^{-3}, R_{\mathrm{b}}=2.910^{15} \rightarrow 3.110^{15} \mathrm{~cm}, \gamma_{\text {cut }}=$ $410^{6} \rightarrow 10^{7}$ ). The assumed level of this radiation is relatively weak in the blob frame and can not modify too much the total level of the observed $\mathrm{TeV}$ radiation. However, it can significantly modify the observed spectral shape (see Fig. 4). The currently available data cannot help us to distinguish if such external radiation field exists in Mrk 501. Observations in the sub- $\mathrm{TeV}$ range appear mandatory to further constrain the influence of external thermal radiation. Low-energy atmospheric Cerenkov telescopes (Ong 1998) such as the gamma-ray telescopes CELESTE (De Naurois 2000) and STACEE (Covault et al. 1999) which recently started operation, are dedicated to work in this energy. They should provide important data for investigations of the external radiation fields in blazars.

\subsection{Temporal evolution}

Up to now, we showed how our simple scenario can fit spectral shapes at different activity states. This section illustrates how to apply the model to multi-frequency light curves. To start a temporal analysis of the high energy flux variations of Mrk 501 during active state, we combine data from different instruments which observed the source in April 1997. We use the X-ray data from two satellites, the All-Sky Monitor (ASM) on the Rossi X-Ray Timing Explorer, and the Oriented Scintillation Spectrometer Experiment (OSSE) on the Compton Gamma Ray Observatory. The VHE observations come from the Whipple a ground based telescope (Catanese et al. 1997). Additional information were derived from previously mentioned observations made by BeppoSAX and CAT experiments. These data provide 
light curves of Mrk 501 with a rather good temporal coverage.

As a first step we assume a simple linear evolution in time of parameters $\left(K_{1}, \gamma_{\mathrm{b}}, R_{\mathrm{B}}\right)$ derived in Sect. 4.1 (see Table 2) to check how this simple modeling can reproduce the observed light curves. It results in a nonlinear temporal evolution of the emitted fluxes and it can roughly reproduce the data points gathered by ASM/XTE. Initially the X-ray radiation increases due to growth of the $K_{1}$ and $\gamma_{\mathrm{b}}$ but after a few days large values of $\gamma_{\mathrm{b}}$ efficiently shift the peak of the synchrotron radiation towards highest energies. This causes decrease of the emission at the frequencies observed by ASM/XTE.

However, this simple modeling does not provide a precise description of the state of Mrk 501 between April 7th and 16th. Especially an intermediate state, observed simultaneously by BeppoSAX and CAT experiments on April 11th, is not at all reproduced by such linear interpolation. More sophisticated but still monotonous interpolations between these two dates appear also unable to fit the high energy spectrum observed on April 11th and do not generate satisfactory light curves at the different X-rays and gamma-rays during April 1997. Therefore the two active events detected on April 7th and 16th are somewhere independent one from the other, although they both belong to the global increase of activity of Mrk 501 over a few months, as shown by the ASM/XTE data.

The available light curves suggest the presence of time scale variability much shorter than 10 days. Short time scales are also deduced from theoretical consideration. For instance, assuming only synchrotron cooling, it can be seen from Eq. (26), that efficiency of the process for high energy particles should be quite high within our scenario. Particles with Lorentz factor of about $\gamma \sim 210^{6}$ are cooled to Lorentz factors of the order of $\gamma \sim 210^{5}$ within 25 hours in the blob frame. All this suggests that, short time scale events occurred between April 7th and 16th, and that the temporal evolution of the source is rather better described by few separate flares during this ten days than by one "slowly" evolving high state.

We therefore introduce two time scales to reproduce the temporal evolution. We assume variation in time for three parameters, namely $R_{\mathrm{b}}, K_{1}$ and $\gamma_{\mathrm{b}}$ as in Sect. 4.1 and use the Gaussian functions as convenient approximation to simulate these changes. The radius $R_{\mathrm{b}}$ and the particle density $K_{1}$ evolve slowly on time scale of ten days, with a minimum of $R_{\mathrm{b}}$ and a maximum of $K_{1}$ on April 16th:

$$
\begin{aligned}
& K_{1}(t)=10_{\mathrm{f}}^{3}+2.410_{\mathrm{f}}^{3} \exp \left[-\frac{\left(t-t_{3}\right)^{2}}{2.5}\right] \\
& R_{\mathrm{b}}(t)=4.210_{\mathrm{f}}^{15}-1.310_{\mathrm{f}}^{15} \exp \left[-\frac{\left(t-t_{3}\right)^{2}}{2.5}\right],
\end{aligned}
$$

where the f indexed values are constrained from our high energy fits. The times of the flares are constrained from the observed light curves, $t_{1}=50548.4, t_{2}=50551.2$, $t_{3}=50554.4$ defined in Modified Julian Days (MJD).

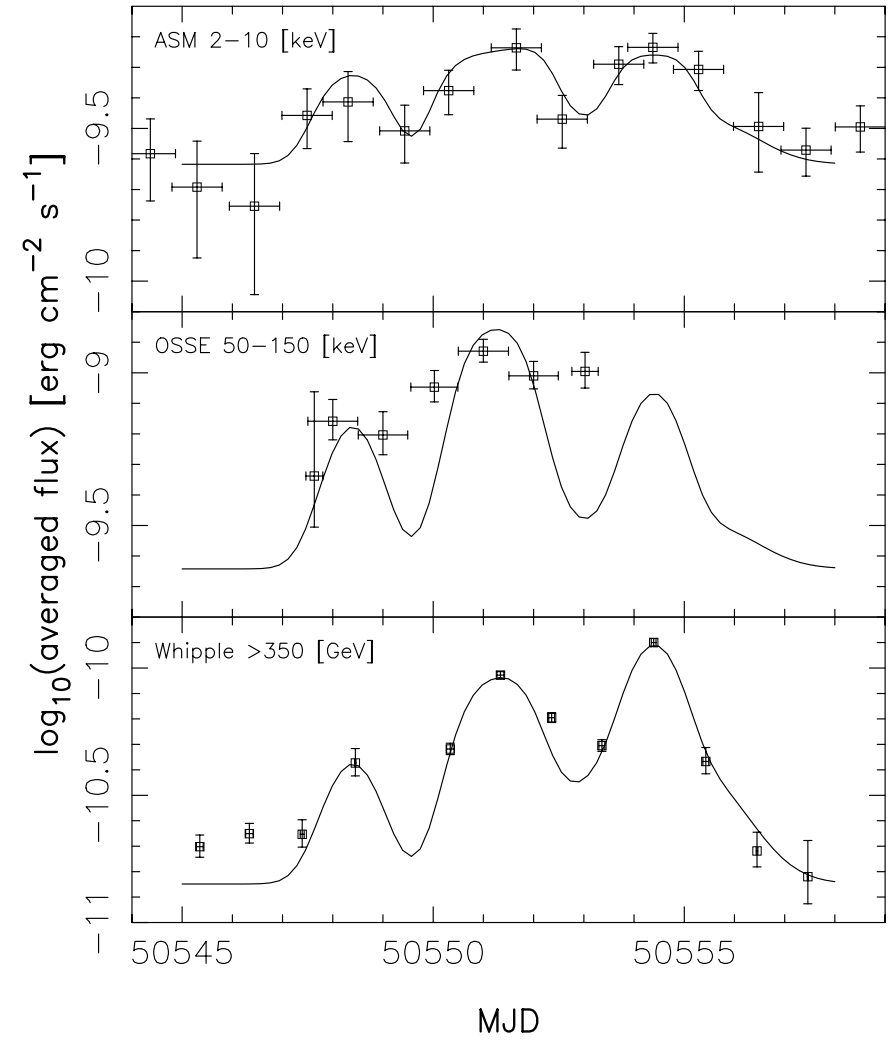

Fig. 5. Multi-frequency variability of Mrk 501 at the time of the two states observed by BeppoSAX and CAT experiments (medium state of April 7th is at MJD $=50545$, high state of April 16th is at MJD = 50 554). The data points have been obtained by three different instruments in the X-rays and gammarays bands (Catanese et al. 1997). Theoretical light curves have been calculated from the SSC model, by integrating the generated spectra over the different observational bands (see text)

The break in electrons energy spectrum $\gamma_{\mathrm{b}}$, evolves on a shorter time scale of about one day, with three peaks from April 7th (50545 MJD) to 16th (50554 MJD), corresponding to three observed flares:

$$
\begin{aligned}
\gamma_{\mathrm{b}}(t)=2 & 2.210_{\mathrm{f}}^{5} \\
& +5.0010^{5} \exp \left[-\frac{\left(t-t_{1}\right)^{2}}{0.5}\right] \\
& +1.7610^{6} \exp \left[-\frac{\left(t-t_{2}\right)^{2}}{0.7}\right] \\
& +6.3010_{\mathrm{f}}^{5} \exp \left[-\frac{\left(t-t_{3}\right)^{2}}{0.45}\right] .
\end{aligned}
$$

Indeed the two major flares around $t_{2}$ and $t_{3}$ are quite well visible on ASM/XTE and Whipple light curves. Existence of another flare around $t_{1}$ is confirmed by spectral observations made by CAT and BeppoSAX around April 11th. The observed spectral fluxes were only slightly higher than on April 7th, while data obtained with other instruments show significant increase of the radiation between these two dates.

We constrain values of the physical parameters at two epochs from our previous fits of the spectral energy distribution. Calculations are made for SSC model with absorption due to pair production inside the source and due to 
interaction with the infrared background in the intergalactic medium. Finally we get good reproduction for ASM and the Whipple data and reasonable correlation with the OSSE observations (see Fig. 5). The flares that we characterize here well give account for specific features of the three light curves such as smaller amplitude of variation at $2-10 \mathrm{keV}$, and a third peak clearly higher in the GeV$\mathrm{TeV}$ range than in the lower frequency ranges. They are likely the signature of sudden acceleration or injection of relativistic particles induced by intermittent shocks, turbulence, or magnetic reconnection in the medium.

The approach developed in this section has many free parameters and we do not claim that the solution we propose here is the unique one. However, it gives an example of the complexity of the events which occurred in Mrk 501 during twelve active days in April 1997. It also illustrates that the small flux variations seen on the different light curves can be well described by SSC model over several orders of magnitude in frequency. The best description is obtained by adding one single slowly evolving high state, with an additional series of flares superposed on it.

\section{Summary}

In this paper, we have build a "blob-in-jet" model which very well describes the multiwavelength emission of Mrk 501. At least three components appear necessary to account for the main characteristics of the observed spectral energy distribution, namely a radio jet, a relativistic plasma blob inside the jet, and the stellar population of the host galaxy. We show how these three components can be described by realistic physical parameters and how a very simple and complete picture of the whole source can emerge from them.

SSC emission from the blob appears able to fit the high energy radiation of Mrk 501 and to explain simultaneous variation seen in X-rays and gamma-rays. Moreover, there is no clear evidence for the presence of strong external radiation field, which suggests that external Compton emission is not dominant. We therefore worked in this frame assuming SSC radiation from a homogeneous spherical blob embedded in the jet and derived basic constraints for the physical parameters describing the blob and its particle energy distribution.

The emission of the source from the ultraviolet wavelengths up to very high energy gamma-rays is well reproduced by this SSC scenario. The spectral shapes of such emission are directly related to the spectral energy distribution of the emitting electrons. To fit the spectral shapes of the data observed by BeppoSAX, we use broken power law functions with the sharp cut-off instead of simple broken power law previously considered (e.g. Pian et al. 1998; Tavecchio et al. 1998). It allows us to fit very well the tail of the $\mathrm{TeV}$ spectrum and the X-ray spectrum as well.The two high states of Mrk 501, observed in April 1997, appear to present a different relative efficiency of the inverseCompton scattering. We show the possibility to fit these two high states (April 7th and 16th) with exactly the same values of all parameters except two parameters related to the particle energy distribution (break $\gamma_{\mathrm{b}}$ and density $K_{1}$ ) and one of the three parameters related to macroscopic blob properties $\left(R_{\mathrm{b}}, B\right.$, or $\left.\delta_{\mathrm{b}}\right)$.

Synchrotron radiation from a conical jet filled by relativistic electrons and magnetic field, with particle density and magnetic field decreasing with distance from the central engine, very well gives account for the observed emission from the low radio frequencies up to the ultraviolet wavelengths. We show how to constrain the most important physical parameters of the inhomogeneous jet from high energy data and calculations performed within a simple homogeneous SSC model, which is a new approach of the problem. Other parameters characterizing the jet can be estimated from the available high angular resolution radio maps, from the estimated mass of the black hole and from a typical size of the accretion disc in the host galaxy.

We tested the influence of weak external radiation fields for inverse-Compton scattering in the blob frame. We found that external synchrotron photons from the jet scattered by high energy electrons from the blob can provide also very good fits for the VHE radiation of blazars. This may be an interesting aspect of the "blob-in-jet" scenario. Additional weak thermal external radiation cannot alone explain the VHE emission but can modify significantly the observed gamma-ray spectra and induce changes in the physical parameters derived from pure SSC models.

The effect of internal absorption is almost negligible in the range of admissible parameters. The intergalactic absorption appears more efficient and can modify the spectral shape and the total flux estimates at highest energies.

We succeeded to characterize physical parameters of the flares responsible for the time evolution of the X-ray and the gamma-ray light curves during activity period in April 1997. The observed flares were reproduced mainly by variation of the break in the electrons spectrum. The time scale for this variability is about a few hours in the observer's frame. Such flaring events can be produced for instance by acceleration or fast injection of high energetic electrons into the emitting region. However, to reproduce more accurately the light curves we had to assume also a change of the radius $\left(R_{\mathrm{b}}\right)$ and the particle density $\left(K_{1}\right)$ on longer time scale. Especially the flares observed around 13th and 16th April cannot be explained by variation of the electrons spectrum only. This remains in good agreement with our previous fits of the spectra from April 7th and 16 th. The variation of $K_{1}$ and $R_{\mathrm{b}}$ which we suggest have time scale of about two days and can be explained for instance as a slow compression of the emitting region.

Acknowledgements. We thank B. Rocca-Volmerange for help with running the galaxy evolution model PEGASE and M. Hanasz for discussion during the first stage of this work. We are grateful to E. Pian, A. Djannati-Atai, M. Catanese and J. Kataoka for data obtained from BeppoSAX, CAT, Whipple OSSE and EGRET experiments and to B. Degrange and the CAT team for discussion. K. Katarzyński thanks the DAEC 
and the DARC of the Paris-Meudon Observatory for discussion and hospitality.

This project was partially supported by the "Réseau Formation-Recherche" and the "Jumelage" for French-Polish collaboration, from the French Minister of Research, Education and Technology.

\section{Appendix A: Approximation of the synchrotron emission coefficient and the electron self-absorption coefficient}

To simplify numerical calculations of the SSC emission of electrons with the power law energy distribution we approximate the mean emission coefficient for a single electron (Crusius \& Schlickeiser 1986; Ghisellini et al. 1988; Chiaberge \& Ghisellini 1999 see their Eq. (13)). From the least square fitting we obtain:

$$
\begin{aligned}
& P_{\mathrm{e}}\left(\nu_{\mathrm{s}}^{\prime}, \gamma\right) \sim \frac{3 \sqrt{3} \sigma_{\mathrm{T}} c U_{B}}{\pi \nu_{B}} c_{1} t^{c_{2}} \exp \left(-c_{3} t\right), \\
& U_{B}=\frac{B^{2}}{8 \pi}, \quad t=\frac{\nu_{\mathrm{s}}^{\prime}}{3 \gamma^{2} \nu_{\mathrm{B}}}, \quad \nu_{B}=\frac{e B}{2 \pi m_{\mathrm{e}} c},
\end{aligned}
$$

where $B$ is the magnetic field intensity $c_{1}=0.78, c_{2}=$ $0.25, c_{3}=2.175$. Now, we can rewrite the synchrotron emission coefficient (2) for any energy distribution of electrons:

$$
\begin{aligned}
j_{\mathrm{s}}^{\prime}\left(\nu_{\mathrm{s}}^{\prime}\right)= & \frac{9 \sigma_{\mathrm{T}} c U_{B} c_{1}}{24 \pi^{2} \nu_{B}} \sqrt{\frac{\nu_{\mathrm{s}}^{\prime}}{\nu_{B}}} \int_{t_{\mathrm{min}}}^{t_{\mathrm{cut}}} N_{\mathrm{e}}\left(\sqrt{\frac{\nu_{\mathrm{s}}^{\prime}}{3 t \nu_{B}}}\right) \\
& \times t^{c_{2}-\frac{3}{2}} \exp \left(-c_{3} t\right) \mathrm{d} t
\end{aligned}
$$

where

$$
t_{\min }=\frac{\nu_{\mathrm{s}}^{\prime}}{3 \gamma_{\min }^{2} \nu_{B}}, \quad t_{\text {cut }}=\frac{\nu_{\mathrm{s}}^{\prime}}{3 \gamma_{\text {cut }}^{2} \nu_{B}} .
$$

This equation can be easily integrated numerically. However, for power-law distribution it can be integrated analytically according to formula:

$$
\begin{aligned}
\int_{x_{1}}^{x_{2}} x^{a} \exp (-b x) \mathrm{d} x= & -x_{2}^{1+a}\left(b x_{2}\right)^{-a-1} \Gamma\left(1+a, b x_{2}\right) \\
& +x_{1}^{1+a}\left(b x_{1}\right)^{-a-1} \Gamma\left(1+a, b x_{1}\right),
\end{aligned}
$$

where $\Gamma$ is the incomplete gamma function. In our case where the electron energy spectrum is given by a power law with a break and a cut-off, we can write

$$
\begin{aligned}
j_{\mathrm{s}}^{\prime}\left(\nu_{\mathrm{s}}^{\prime}\right)= & \frac{9 \sigma_{\mathrm{T}} c U_{B} c_{1}}{24 \pi^{2} \nu_{B}} \sqrt{\frac{\nu_{\mathrm{s}}^{\prime}}{\nu_{B}}} \\
& \times\left\{g\left(\nu_{\mathrm{s}}^{\prime}, t_{\mathrm{b}}, n_{1}, K_{1}\right)-g\left(\nu_{\mathrm{s}}^{\prime}, t_{\mathrm{min}}, n_{1}, K_{1}\right)\right. \\
& \left.+g\left(\nu_{\mathrm{s}}^{\prime}, t_{\text {cut }}, n_{2}, K_{2}\right)-g\left(\nu_{\mathrm{s}}^{\prime}, t_{\mathrm{b}}, n_{2}, K_{2}\right)\right\}
\end{aligned}
$$

where

$$
\begin{aligned}
g\left(\nu_{\mathrm{s}}^{\prime}, t, n, K\right)= & K\left(\frac{\nu_{\mathrm{s}}^{\prime}}{3 \nu_{B}}\right)^{\frac{-n}{2}} t^{c_{2}+\frac{n-1}{2}}\left(c_{3} t\right)^{\frac{1-n}{2}-c_{2}} \\
& \times \Gamma\left(c_{2}+\frac{n-1}{2}, c_{3} t\right), t_{\mathrm{b}}=\frac{\nu_{\mathrm{s}}^{\prime}}{3 \gamma_{\mathrm{b}}^{2} \nu_{B}}
\end{aligned}
$$

Within formula (A.1) for the single electron emissivity and for the spectrum of the electron energy described by Eq. (1) we can integrate also the absorption coefficient:

$$
\begin{aligned}
k^{\prime}\left(\nu_{\mathrm{s}}^{\prime}\right)=\frac{3}{3} \sigma_{\mathrm{T}} c U_{B} c_{1} \\
16 \pi^{2} m_{\mathrm{e}} \nu_{\mathrm{s}}^{\prime 2} \nu_{B} \\
\quad \times\left\{h\left(\nu_{\mathrm{s}}^{\prime}, t_{\mathrm{b}}, n_{1}, K_{1}\right)-h\left(\nu_{\mathrm{s}}^{\prime}, t_{\mathrm{min}}, n_{1}, K_{1}\right)\right. \\
\left.\quad+h\left(\nu_{\mathrm{s}}^{\prime}, t_{\mathrm{cut}}, n_{2}, K_{2}\right)-h\left(\nu_{\mathrm{s}}^{\prime}, t_{\mathrm{b}}, n_{2}, K_{2}\right)\right\}
\end{aligned}
$$

where

$$
\begin{aligned}
h\left(\nu_{\mathrm{s}}^{\prime}, t, n, K\right)= & K(n+2) 3^{\frac{n}{2}}\left(\frac{\nu_{\mathrm{s}}^{\prime}}{\nu_{B}}\right)^{\frac{-n}{2}} t^{c_{2}+\frac{n}{2}} \\
& \times\left(c_{3} t\right)^{-c_{2}-\frac{n}{2}} \Gamma\left(c_{2}+\frac{n}{2}, c_{3} t\right) .
\end{aligned}
$$

We can compare the approximated coefficients used here with two other approaches, first the one by Blumenthal \& Gould (1970) and Ghisellini et al. (1985), and second the one by Jones et al. (1974) and Band \& Grindlay (1985) for a range of values for the physical parameters with $B$ from $10^{-3}$ to $10^{3} \mathrm{G}, K$ from 1 to $10^{5} \mathrm{~cm}^{-3}, n$ from 1.5 to 4 . The differences which appear between the three methods are of the order of $1 \%$, or even less. Therefore the influence of the approximation on the resulting SSC spectra is completely negligible.

\section{Appendix B: Position of the inverse-Compton peak}

To find the position of the frequency peak in the inverseCompton spectrum, we adopt the approximated version of TMG formula for the inverse-Compton emissivity:

$$
\begin{aligned}
\epsilon_{\mathrm{c}}\left(x_{\mathrm{c}}^{\prime}\right)= & \frac{\sigma_{\mathrm{T}} m_{\mathrm{e}} c^{3}}{8 \pi h} \int_{x_{\mathrm{s}_{1}}^{\prime}}^{x_{\mathrm{s}_{2}}^{\prime}} N_{\mathrm{e}}\left(\sqrt{\frac{3.0 x_{\mathrm{c}}^{\prime}}{4.0 x_{\mathrm{s}_{0}}^{\prime}}}\right) \sqrt{\frac{3.0 x_{\mathrm{c}}^{\prime}}{4.0 x_{\mathrm{s}_{0}}^{\prime}}} \\
& \times \frac{u_{\mathrm{r}}\left(x_{\mathrm{s}_{0}}^{\prime}\right)}{x_{\mathrm{s}_{0}}^{\prime}} \mathrm{d} x_{\mathrm{s}_{0}}^{\prime},
\end{aligned}
$$

where $u_{\mathrm{r}}$ is the synchrotron radiation field density and $x^{\prime}=h \nu^{\prime} / m_{\mathrm{e}} c$ (TMG). For a broken power-law particle energy spectrum with the cut-off at high energies, the radiation field density can be also approximated as a broken power-law function with a cut-off at high frequencies. Under the above assumptions, the inverse-Compton emissivity can be split into four simple integrals. However, taking into account Klein-Nishina restrictions for the high energy part of the spectrum it is very easy to show that only components produced by low energy synchrotron photons, 
and the electrons with different energies are important. The equations which describe this radiation are identical to those given by TMG (see their Eqs. (A5), (A6), (A12), and (A13), with $\ln \left(x_{1} / x_{2}\right)$ corrected to $\ln \left(x_{2} / x_{1}\right)$ in (A5)). This expression of the inverse-Compton emissivity in the Klein-Nishina limit is still too complex to be directly used in the estimation of the inverse-Compton peak frequency. Following the method used by TMG we can write formula similar to their Eq. (A16):

$$
\begin{aligned}
\epsilon_{\text {peak }}\left(x_{\mathrm{c}}^{\prime}\right)= & \frac{\sigma_{\mathrm{T}} m_{\mathrm{e}} c^{3}}{8 \pi h}\left[\left(\frac{4}{3}\right)^{-\alpha_{1}} K_{1} u_{\mathrm{r}_{0}} x_{\mathrm{c}}^{\prime-\alpha_{1}} \ln \left(\frac{\gamma_{\mathrm{b}}^{2}}{x_{\mathrm{c}}^{\prime 2}}\right)\right. \\
& +\left(\frac{4}{3}\right)^{-\alpha_{2}} \frac{K_{2} u_{\mathrm{r}_{0}} x_{\mathrm{c}}^{\prime-\alpha_{2}}}{\alpha_{2}-\alpha_{1}}\left(\left\{\frac{3 x_{\mathrm{c}}^{\prime}}{4 \gamma_{\mathrm{b}}^{2}}\right\}^{\alpha_{2}-\alpha_{1}}\right. \\
& \left.\left.-\left\{\frac{3 x_{\mathrm{c}}^{\prime}}{4 \gamma_{\text {cut }}^{2}}\right\}^{\alpha_{2}-\alpha_{1}}\right)\right] .
\end{aligned}
$$

In the second part of this formula we use full version of the Eq. (A12) (TMG) instead the part which comes only from the upper boundary condition used by TMG. This is necessary because in our assumption for the high energy states the parameter $\gamma_{\text {cut }}$ may be not significantly greater than $\gamma_{\mathrm{b}}$.

We then deduce the position of the inverse-Compton peak frequency as the maximum of the function $x_{\mathrm{c}}^{\prime} \epsilon_{\mathrm{peak}}\left(x_{\mathrm{c}}^{\prime}\right)$,

$$
\begin{aligned}
x_{\mathrm{c}_{\text {peak }}}^{\prime}= & \gamma_{\mathrm{b}} \exp \left(\frac{1-\gamma_{\mathrm{cut}}^{2\left(\alpha_{1}-\alpha_{2}\right)} \gamma_{\mathrm{b}}^{2\left(\alpha_{2}-\alpha_{1}\right)}-2 \alpha_{2}}{2\left(\alpha_{1}-1\right)\left(\alpha_{1}-\alpha_{2}\right)}\right. \\
& \left.+\frac{\alpha_{1}+\alpha_{1} \gamma_{\mathrm{cut}}^{2\left(\alpha_{1}-\alpha_{2}\right)} \gamma_{\mathrm{b}}^{2\left(\alpha_{2}-\alpha_{1}\right)}}{2\left(\alpha_{1}-1\right)\left(\alpha_{1}-\alpha_{2}\right)}\right) \\
= & \gamma_{\mathrm{b}} \exp \left[\frac{1}{\alpha_{1}-1}+\frac{1-\left(\frac{\gamma_{\mathrm{b}}}{\gamma_{\mathrm{cut}}}\right)^{2\left(\alpha_{2}-\alpha_{1}\right)}}{2\left(\alpha_{2}-\alpha_{1}\right)}\right] .
\end{aligned}
$$

The expression is valid only for the Klein-Nishina limit. Its domain of validity is given in terms of the synchrotron break frequency, $\nu_{\mathrm{s}_{\mathrm{b}}}^{\prime}$,

$\frac{3}{4} \frac{m_{\mathrm{e}} c^{2}}{h} \frac{1}{\gamma_{\mathrm{b}}}<\nu_{\mathrm{s}_{\mathrm{b}}}^{\prime}<\frac{3}{4} \frac{m_{\mathrm{e}} c^{2}}{h} \frac{1}{\gamma_{\min }}$.

Equation (B.5) provides a simple relation between $\gamma_{\mathrm{b}}$ and $\gamma_{\text {cut }}$,

$\gamma_{\mathrm{b}}=\gamma_{\text {cut }} \sqrt{\frac{C_{\mathrm{p}} \nu_{\mathrm{s}_{\mathrm{b}}}}{C_{\mathrm{b}} \nu_{\mathrm{s}_{\mathrm{p}}}}}$,

where $C_{\mathrm{b}}=3.710^{6}$ and $C_{\mathrm{p}}$ is given by Eq. (28). Inserting this formula to Eq. (B.3) we obtain a direct relation between $\gamma_{\text {cut }}$ and observable quantities.

\section{Appendix C: The total inverse-Compton flux}

Using the inverse-Compton emissivity (B.1) we estimate the total inverse-Compton flux by taking into account its two dominant components discussed previously. To avoid inconvenience of $\max \& \min$ functions in the definitions of the components (Eqs. (A6), (A13) in TMG) we split the inverse-Compton spectrum at positions where the boundary conditions are changing. The dominant part of the inverse-Compton emission can be then described by:

$F_{\mathrm{c}}\left(\nu_{\mathrm{c}}\right) \simeq\left\{\begin{array}{l}C_{x} f_{1}\left(\nu_{\mathrm{c}}\right), \nu_{\mathrm{c}_{1}} \leq \nu_{\mathrm{c}}<\nu_{\mathrm{c}_{2}} \\ C_{x} f_{2}\left(\nu_{\mathrm{c}}\right), \nu_{\mathrm{c}_{2}} \leq \nu_{\mathrm{c}}<\nu_{\mathrm{c}_{3}} \\ C_{x} f_{3}\left(\nu_{\mathrm{c}}\right), \nu_{\mathrm{c}_{3}} \leq \nu_{\mathrm{c}}<\nu_{\mathrm{c}_{4}},\end{array}\right.$

where

$$
\begin{aligned}
f_{1}\left(\nu_{\mathrm{c}}\right) & =Q_{1} \nu_{\mathrm{c}}^{-\alpha_{1}} \ln \left(\frac{Q 2}{\nu_{\mathrm{c}}}\right)+Q_{3}\left(Q_{4}-Q_{5}\right) \nu_{\mathrm{c}}^{-\alpha_{1}} \\
f_{2}\left(\nu_{\mathrm{c}}\right) & =R_{1} \nu_{\mathrm{c}}^{-\alpha_{1}} \ln \left(\frac{R_{2}}{\nu_{\mathrm{c}}^{2}}\right)+R_{3}\left(R_{4}-R_{5}\right) \nu_{\mathrm{c}}^{-\alpha_{1}} \\
f_{3}\left(\nu_{\mathrm{c}}\right) & =S_{1}\left(S_{2} \nu_{\mathrm{c}}^{\alpha_{1}-2 \alpha_{2}}-S_{3} \nu_{\mathrm{c}}^{-\alpha_{1}}\right) \\
Q_{1} & =R_{1}=\left(\frac{3(1+z) h}{4 m_{\mathrm{e}} c^{2} \delta_{\mathrm{b}}}\right)^{-\alpha_{1}}, \\
Q_{2} & =\frac{4}{3} \nu_{\mathrm{s}_{\mathrm{b}}} \gamma_{\mathrm{b}}^{2} \\
Q_{3} & =R_{3}=S_{1}=\frac{\gamma_{\mathrm{b}}^{n_{2}-n_{1}}}{\alpha_{2}-\alpha_{1}}\left(\frac{3(1+z) h}{4 m_{\mathrm{e}} c^{2} \delta_{\mathrm{b}}}\right)^{-\alpha_{2}} \\
Q_{4} & =R_{4}=\left(\frac{3(1+z) h}{4 m_{\mathrm{e}} c^{2} \delta_{\mathrm{b}} \gamma_{\mathrm{b}}^{2}}\right)^{\alpha_{2}-\alpha_{1}} \\
Q_{5} & =R_{5}=S_{3}=\left(\frac{3(1+z) h}{4 m_{\mathrm{e}} c^{2} \delta_{\mathrm{b}} \gamma_{\mathrm{cut}}^{2}}\right)^{\alpha_{2}-\alpha_{1}} \\
R_{2} & =\frac{m_{\mathrm{e}}^{2} c^{4} \delta_{\mathrm{b}}^{2} \gamma_{\mathrm{b}}^{2}}{(1+z)^{2} h^{2}}, \\
S_{2} & =\left(\frac{3}{4} \frac{\delta_{\mathrm{b}} m_{\mathrm{e}} c^{2}}{h(1+z)}\right)^{\alpha_{2}-\alpha_{1}} .
\end{aligned}
$$

The frequencies are given by:

$$
\begin{aligned}
& \nu_{\mathrm{c}_{1}}=\frac{4}{3} \gamma_{\min }^{2} \nu_{\mathrm{s}_{\mathrm{b}}} \\
& \nu_{\mathrm{c}_{2}}=\frac{3}{4}\left(\frac{\delta_{\mathrm{b}}}{1+z} \frac{m_{\mathrm{e}} c^{2}}{h}\right)^{2} \frac{1}{\nu_{\mathrm{s}_{\mathrm{b}}}} \\
& \nu_{\mathrm{c}_{3}}=\frac{\delta_{\mathrm{b}}}{1+z} \frac{m_{\mathrm{e}} c^{2}}{h} \gamma_{\mathrm{b}}, \\
& \nu_{\mathrm{c}_{4}}=\frac{\delta_{\mathrm{b}}}{1+z} \frac{m_{\mathrm{e}} c^{2}}{h} \gamma_{\text {cut }} .
\end{aligned}
$$

The value of the constant $C_{x}$ can be easily obtained from the relation:

$C_{x}=\frac{F_{\mathrm{c}}\left(\nu_{\mathrm{c}_{\mathrm{peak}}}\right)}{f_{2}\left(\nu_{\mathrm{c}_{\mathrm{peak}}}\right)}$

The total inverse-Compton flux can finally be calculated by analytical integration of Eq. (C.1). 


\section{References}

Aharonian, F. A., Akhperjanian, A. G., Barrio, J. A., et al. 1999, A\&A, 327, L5

Aharonian, F. A. 2000, New Astr., 5, 377

Band, D. L., \& Grindlay, J. E. 1985, ApJ, 289, 128

Bednarek, W., \& Protheroe, R. J. 1997, MNRAS, 292, 646

Bednarek, W., \& Protheroe, R. J. 1999, MNRAS, 310, 577

Bloom, S. D., \& Marscher, A. P. 1996, ApJ, 461, 657

Blumenthal, G. R., \& Gould, R. J. 1970, RvMP, 42, 237

Bottcher, M., \& Bloom, S. D. 2000, ApJ, 119, 469

Catanese, M., Bradbury, S. M., Breslin, A. C., et al. 1997, ApJ, 487, L143

Catanese, M., \& Weeks, T. C. 1999, PASP, 111, 1193

Celotti, A., Maraschi, L., \& Treves, A. 1991, A\&A, 377, 403

Chiaberge, M., \& Ghisellini, G. 1999, MNRAS, 306, 551

Conway, J. E., \& Wrobel, J. M. 1995, ApJ, 439, 98

Coppi, P. S., \& Blandford, R. D. 1990, MNRAS, 245, 453

Coppi, P. S., Kartje, T. F. \& Königl, A. 1993, Proc. Compton Symp., AIP

Covault, C. E., Chantell, M. C., Conner, Z., et al. 1999, AAS, 194, 0907

Crusius, A., \& Schlickeiser, R. 1986, A\&A, 164, L16

De Naurois, M. 2000, Ph.D. Thesis, Paris VI University

Dermer, C. D. 1999, APh, 11, 1

Dermer, C. D., \& Schlickeiser, R. 1993, ApJ, 416, 458

Djannati-Atai, A., Piron, F., Barrau, A., et al. 1999, A\&A, 350,17

Fioc, M., \& Rocca-Volmerange, B. 1997, A\&A, 326, 950

Gear, W. K. 1993, MNRAS, 264, L21

Gear, W. K., Stevens, J. A., Hughes, D. H., et al. 1994, MNRAS, 267, 167

Ghisellini, G., Maraschi, L., \& Treves, A. 1985, A\&A, 146, 204

Ghisellini, G., \& Madau, P. 1996, MNRAS, 280, 67

Ghisellini, G., Guilbert, P., \& Svensson, R. 1988, ApJ, 334, L5

Ghisellini, G. 1998, in Proc. Active X-ray Sky Symp., Rome, Italy

Ghisellini, G. 1999, APh, 11, 11

Gould, R. J. 1979, A\&A, 76, 306

Guy, J., Renault, C., Aharonian, F. A., et al. 2000, A\&A, 359, 419

Hughes, P. A., Aller, H. D., \& Aller, M. F. 1989a, ApJ, 341, 54 ,

Hughes, P. A., Aller, H. D., \& Aller, M. F. 1989b, ApJ, 341, 68

Impey, C., \& Gregorini, L. 1993, AJ, 105, 853

Inoue, S., \& Takahara, F. 1996, ApJ, 463, 555

Jones, F. C. 1968, Phys. Rev., 167, 1159

Jones, T. W., O’Dell, S. L., \& Stein, W. A. 1974, ApJ, 188, 353

Kardashev, N. S. 1962, Soviet Astron., AJ, 6, 317
Kataoka, J., Mattox, J. R., Quinn, J., et al. 1999, ApJ, 514, 138

Kirk, J. G., Rieger, F. M., \& Mastichiadis, A. 1998, A\&A, 333, 452

Konigl, A. 1981, ApJ, 243, 700

Kormendy, J., \& Richstone, D. 1995, ARA\&A, 33, 581

Lawrence, A., Rowan-Robinson, M., Efstathiou, A., et al. 1991, MNRAS, 248, 91

Mannheim, K. 1993, A\&A, 269, 67

Malkan, M. A., \& Stecker, F. W. 1998, ApJ, 496, 13

Marscher, A. P. 1980, ApJ, 235, 386

Marscher, A. P., \& Gear, W. K. 1985, ApJ, 289, 114

Melrose, D. B. 1980, Plasma Astrophysics (Gordon and Breach, Science Publishers, Inc.), vol. 2

Mucke, A., \& Protheroe, R. J. 2000 [astro-ph 0004052v3]

Mufson, S. L., Hutter, D. J., Hackney, K. R., et al. 1984, ApJ, 285,571

Nilsson, K., Pursimo, T., Takalo, L. O., et al. 1999, PASP, 111, 1223

Ong, R. A. 1998, Phys. Rep., 305, 93

O'Dell, S. L. 1988, ApJ, 327, 60

Petry, D., Bradbury, S. M., Konopelko, A., et al. 1996, A\&A, 311L, 13

Pian, E., \& Treves, A. 1993, ApJ, 416, 130

Pian, E., Vacanti, G., Tagliaferri, G., et al. 1998, ApJ, 492, L17

Pohl, M., \& Schlickeiser, R. 2000, A\&A, 354, 395

Punch, M., Akerlof, C. W., Cawley, M. F., et al. 1992, Nature, 358,477

Quinn, J., Akerlof, C. W., Biller, S., et al. 1996, ApJ, 456, L83

Quinn, J., Bond, I. H., Boyle, P. J., et al. 1999, ApJ, 518, 693

Rybicki, G., \& Lightman, A. P. 1979, Radiative Processes in Astrophysics (Wiley Interscience, New York)

Samuelson, F. W., Biller, S. D., Bond, I. H., et al. 1998, ApJ, 501L, 17

Sikora, M., Begelman, M. C., \& Rees, M. J. 1994, ApJ, 421, 153

Sikora, M., Sol, H., Begelman, M. C., et al. 1996, MNRAS, 280,781

Stecker, F. W., \& De Jager, O. C. 1998, A\&A, 334, L85

Steppe, H., Salter, C. J., Chini, R., et al. 1988, A\&AS, 75, 317

Takahashi, T., Tashiro, M., Madejski, G., et al. 1996, ApJ, $470 \mathrm{~L}, 89$

Tavecchio, F., Maraschi, L., \& Ghisellini, G. 1998, ApJ, 509, 608 (TMG)

Tavecchio, F., \& Maraschi, L. 2000, in Proc. X-Ray Astronomy '99 [astro-ph/0002431]

van Breugel, W., \& Schilizzi, R. 1986, ApJ, 301, 834

Wiren, S., Valtaoja, E., Terasranta, H., et al. 1992, AJ, 104, 1009

Xie, G. Z., Li, K. H., Zhang, X., et al. 1999, ApJ, 522, 846

Zweerink, J. A., Akerlof, C. W., Biller, S. D., et al. 1997, ApJ, 490L, 141 